\title{
CUT: A multicriteria approach for concavifiable preferences*
}

\author{
Nikolaos Argyris ${ }^{\dagger}$ \\ Department of Statistics, University of Warwick, Coventry, UK. \\ Alec Morton \\ Department of Management Science, Strathclyde Business School, Glasgow, UK. \\ Jose Rui Figueira \\ CEG-IST, Instituto Superior Tecnico, Universidade de Lisboa, Lisbon, Portugal.
}

9 Feb 2014

\begin{abstract}
We consider the problem of helping a decision maker (DM) choose from a set of multiattributed objects when her preferences are "concavifiable", i.e. representable by a concave value function. We establish conditions under which preferences or preference intensities are concavifiable. We also derive a characterization for the family of concave value functions compatible with a set of such preference statements expressed by the DM. This can be used to validate dominance relations over discrete sets of alternatives and forms the basis of an interactive procedure. We report on the practical use of this procedure with several DMs for a flat-choice problem and its computational performance on a set of project-portfolio selection problem instances. The use of preference intensities is found to provide significant improvements to the performance of the procedure.
\end{abstract}

\section{Introduction}

The general problem motivating this paper is that of multicriteria choice, where a decision maker (DM) is faced with selecting from a number of alternatives evaluated on different criteria (or attributes). The alternatives may be too many to list explicitly, or even be infinite in number. Moreover, the DM cannot readily articulate her preferences by specifying a value function, but she can answer specific questions asked of her by an analyst or a decision support system. Such interaction is a learning process, by which the DM reflects, deliberates and constructs her own preferences (see Phillips, 1984; Vanderpooten, 1989, Roy, 1993). This problem has a long history and has been tackled by many researchers over the last several decades.

\footnotetext{
*This is a pre-print of a manuscript accepted for publication in Operations Research.

${ }^{\dagger}$ Corresponding Author: n.argyris@warwick.ac.uk
} 
Perhaps the most fundamental differences among existing approaches relate to their assumptions about the underlying preferential model, e.g. the value function specification and the types of preferential information that the model permits. One family of approaches employ additively separable value functions. Within this family there are clear methodologies for incorporating information about intensity of preference. Another family of approaches employ quasi-concave value functions, based on the assumption of preference-convexity: an average (convex combination) of two alternatives is assumed to be at least as attractive as the least preferred of the two. Within this family it is not standard to use preference intensity information, and indeed it is unclear how one could combine it with the quasi-concavity of the value function. On the other hand, an appealing feature of these approaches is that preference-convexity is in many settings a more natural condition than the preferential-independence conditions which underlie the additive model. Indeed, the quasi-concavity of the value function has come to be viewed as a relatively standard assumption (particularly in the field of economics).

In this paper we introduce an approach for the multicriteria choice problem, for the case where the DM's preferences are concavifiable, i.e. representable by a concave value function. We call our idea CUT, for Concave UTility by analogy with the procedure UTA (Jacquet-Lagrèze and Siskos, 1982), which stands for UTilité Additive. Concavity can be an attractive alternative to both additivity and preference-convexity (though it is closely related to the latter). Firstly, it may be a more natural assumption than additivity in some settings. For example, in the context of buying a house characterized by square footage and distance from the city center, it may be that a DM has concavifiable preferences in the sense that for every level of distance she has diminishing marginal benefit for square footage and vice versa, but nevertheless she values square footage more when she is close to the city center (e.g. because her flat will then be an attractive location for entertaining or hosting visitors). Furthermore, concavifiability is not a particularly stronger assumption than preference-convexity (we elaborate in Section 3), but it can allow for the elicitation and use of preference intensities.

We demonstrate that our method has the following attractive features:

- theoretically, using CUT we can derive all information that can be inferred from a given set of preference statements.

- empirically, asking the DM about preference intensities does improve the effectiveness of an interactive procedure based on CUT, compared to using the same procedure with ordinal preferences only. Crucially, this improvement can be realized without having to ask the DM excessively demanding questions. This demonstrates an important potential for using preference intensities in interactive procedures.

- for the purposes of practical application, our method provides the DM with information on how much better one alternative can be than another, thus giving a measure of the potential cost of prematurely terminating the interactive search.

We structure the paper as follows. In Section 2 we provide a literature review. In Section 3 we introduce our modeling framework; we show that there are two distinct ways to make precise the notion of "concavifiability". In Section 4 we characterize the set of concave value functions compatible with a set of preferential statements and use that to validate dominance relations among a set of alternatives. Section 5 describes the interactive use of CUT: we introduce an interactive procedure and show that it is both practical and has attractive theoretic convergence properties. Section 6 concludes. 


\section{Literature Review}

To frame our contribution, we structure our literature review under the two headings of Multiple Criteria Decision Analysis (MCDA) and Interactive Multiobjective Programming (IMOP). In MCDA, the analyst guides the DM through the construction of an explicit value model. This is exemplified in the framework of Multiattribute Value Theory (Keeney and Raiffa, 1976; Dyer and Sarin, 1979). To obtain an analytically tractable value function, the DM's preferences are assumed to satisfy a separability condition (preference/difference independence, see e.g. Dyer, 2005; Fishburn and Wakker 1995), implying that the value function is of an additive form, or some generalization of this, such as a multilinear or multiplicative form. By far the most common model used in practice is the additive model. In all these models the DM is asked to make choices between objects, or express judgments of preference intensity, as, for example, in the "weighting" question: "How much do you care about the difference between alternatives $a$ and $b$ on criterion 1 , relative to the difference between alternatives $c$ and $d$ on criterion 2?". The concept of preference intensity is pervasive in this framework (see e.g. von Winterfeldt and Edwards, 1986 p. 210, Farquhar and Keller 1989; Keeney and von Winterfeldt, 2007 p. 236) and its use in practice is widely accepted: for example, the concept of "swing-weights" presented in MCDA textbooks (e.g. Belton and Stewart, 2002) is an intensity of preference concept.

Often in practice, DMs find it difficult to provide the precise answers required to parameterize the model, but they may be able to provide other "incomplete information", e.g. ranges for criterion weights, holistic comparisons of alternatives, or qualitative preference intensity statements (see Salo and Hämäläinen, 2010 for a recent survey). Several approaches are based on utilizing such responses to deduce additional preferences and help focus decision maker search and reflection. We highlight those based on the well-known UTA method (Jacquet-Lagrèze and Siskos, 1982) (see Siskos, Grigoroudis and Matsatsinis, 2005, and Greco et al. 2010 for surveys). Predominantly these approaches use additive value functions, although there are exceptions: Greco, Matarazzo and Slowinski (2001) and Angillela, Greco and Matarazzo (2010) consider the use of rough-set and Choquet integral concepts. Nevertheless, how one works with preference information when additivity is not an appropriate assumption is not a settled question in the MCDA literature.

In IMOP approaches the analyst progressively asks DMs to articulate their preferences and uses that to guide the search. As in MCDA, a structural assumption about the value function is typically made. In addition to the additive case (e.g. Stewart, 1987; Jacquet-Lagrèze, Meziani and Slowinski, 1987; Roy et al., 2008), the IMOP literature has also considered other value function specifications. Geoffrion, Dyer, Feinberg (1972) and Zionts and Wallenius (1983) consider the case of a concave value function. When a quasi-concave value function is assumed, a popular idea in IMOP is to use "convex cones" to eliminate entire dominated regions in multiattribute space. Hazen (1983) provides a rigorous analysis of this concept. Korhonen, Wallenius and Zionts (1984), Ramesh, Karwan and Zionts (1988) and Prasad, Karwan and Zionts (1997) provide interactive procedures utilizing convex cones. This idea has proved influential in the literature - Karsu, Morton and Argyris (2012) cite 15 references using cones, a success we attribute to the modest and natural appeal of the quasi-concavity assumption. We note here that IMOP procedures utilizing cones do not allow for deducing all inferable pairwise dominances given a set of preference statements from the DM (see e.g. Hazen, 1983, Korhonen et al. 1996).

In IMOP preference intensities are an unexplored concept: Shin and Ravindran (1991) review eight modes of questioning in IMOP, none of which are based on intensity of preference. Yet, preference intensities can be very useful in an IMOP setting (a claim we justify later in the paper). 
Indeed, Korhonen, Moskowitz and Wallenius (1986) (also Korhonen et al. 1993) suggested the use of measurable value functions in IMOP, to 'allow consideration not only of the question of finding a better solution, but also of "how much better"'. However, there is no way to incorporate preference intensities within the convex cones technology. In principle this is possible within the method of Zionts and Wallenius (1983), but this is only by using a linear approximation of the underlying (quasi-) concave value function (and, as is typically the case, a few ordinal comparisons are already enough to over-determine the approximation).

The approach we introduce, called CUT, combines distinctive advantages of the existing approaches. When used with ordinal preferences, CUT is only slightly less general than approaches based on quasi-concave functions (as we elaborate in the next section); but it has the advantage of accommodating preference intensities as well. CUT allows for capturing all information that can be derived from the DM's expressed preferences (Section 4) and can also be used in an IMOP context (Section 5). Particularly for the IMOP case, utilizing preference intensity information can dramatically improve the effectiveness of an interactive procedure.

\section{Modeling Framework}

We consider a set of outcomes $Z \subseteq \mathbb{R}_{+}^{p}$ and denote an outcome by $z=\left(z^{1}, \ldots, z^{p}\right)$. We assume that $Z$ is convex, contains the origin $\mathbf{0}=(0, \ldots, 0)$ and is not a singleton. The preference relation $\succsim$ orders outcomes in $Z: z^{\prime} \succsim z^{\prime \prime}$ is interpreted as " $z^{\prime}$ is at least as good as $z^{\prime \prime \prime}$. The preference intensity relation (or strength-of-preference relation) $\succsim^{*}$ orders pairs of outcomes in $Z \times Z:\left(z^{\prime} \leftarrow\right.$ $\left.z^{\prime \prime}\right) \succsim^{*}\left(z^{\prime \prime \prime} \leftarrow z^{\prime \prime \prime \prime}\right)$ means that the DM prefers $z^{\prime \prime}$ to $z^{\prime}$ more strongly than she prefers $z^{\prime \prime \prime \prime}$ to $z^{\prime \prime \prime}$, (or feels that exchanging $z^{\prime \prime}$ for $z^{\prime}$ is at least as attractive as exchanging $z^{\prime \prime \prime \prime}$ for $z^{\prime \prime \prime}$ ). The relation $\succsim^{*}$ induces a binary preference relation $\succsim$ on $Z$ as follows: $z^{\prime} \succsim z^{\prime \prime} \Leftrightarrow\left(z^{\prime} \leftarrow z^{\prime \prime}\right) \succsim^{*}$ $\left(z^{\prime \prime} \leftarrow z^{\prime \prime}\right)$. The preference relation $\succsim$ is represented by an ordinal value function $u(\cdot): Z \rightarrow \mathbb{R}$, if: $z^{\prime} \succsim z^{\prime \prime} \Leftrightarrow u\left(z^{\prime}\right) \geq u\left(z^{\prime \prime}\right)$. All functions that represent $\succsim$ are strictly-increasing transformations of one another. The preference intensity relation $\succsim^{*}$ is represented by a cardinal value function, or measurable value function $u(\cdot): z \rightarrow \mathbb{R}$, if: $z^{\prime} \leftarrow z^{\prime \prime} \succsim^{*} z^{\prime \prime \prime} \leftarrow z^{\prime \prime \prime \prime} \Leftrightarrow u\left(z^{\prime}\right)-u\left(z^{\prime \prime}\right) \geq u\left(z^{\prime \prime \prime}\right)-u\left(z^{\prime \prime \prime \prime}\right)$ (note that $u(\cdot)$ will also represent the induced ordinal relation $\succsim$ ). All functions that represent $\succsim^{*}$ are positive linear transformations of one another. We assume that both $\succsim^{*}$ and $\succsim$ satisfy conditions sufficient for the existence of a representing function (these are well known, see e.g. Krantz et al. 1971, Köbberling 2006).

As is commonplace in the literature, we assume that the DM's preferences are weakly monotonic, i.e. $z^{\prime}, z^{\prime \prime} \in Z, z^{\prime \prime} \geq z^{\prime} \Rightarrow z^{\prime \prime} \succsim z^{\prime}$ (where $z^{\prime \prime} \geq z^{\prime}$ means that $z^{\prime \prime}$ is at least as high as $z^{\prime}$ in all $p$ dimensions). Weak monotonicity implies nondecreasingness of the representing value function. We will also require the value function to be concave, i.e. $u\left(\lambda z^{\prime}+(1-\lambda) z^{\prime \prime}\right) \geq \lambda u\left(z^{\prime}\right)+(1-$ $\lambda) u\left(z^{\prime \prime}\right)$ holds for any $z^{\prime}, z^{\prime \prime} \in Z$ and $\lambda \in(0,1)$ (when the inequality is strict the function is strictly concave). There are two ways to approach concavifiability, depending on whether one accepts preference intensities, or ordinal preferences only.

For the ordinal case, the additional condition to be imposed on $\succsim$ is (strict) convexity. Formally, $\succsim$ exhibits (strict) convexity if for all $z^{\prime}, z^{\prime \prime} \in Z, z^{\prime} \succsim z^{\prime \prime} \Rightarrow \lambda z^{\prime}+(1-\lambda) z^{\prime \prime} \succsim z^{\prime \prime}, \lambda \in(0,1)$ (resp. $\lambda z^{\prime}+(1-\lambda) z^{\prime \prime} \succ z^{\prime \prime}$ for strict convexity). A function that represents the (strictly) convex $\succsim$ is called (strictly) quasi-concave. For the case where $Z$ is an infinite set, convexity of $\succsim$ is not sufficiently strong to ensure concavifiability in general (de Finetti, 1949; Fenchel, 1953). However for case of finite $Z$ we may state the following (a special case of a result of Kalandrakis, 2010). 

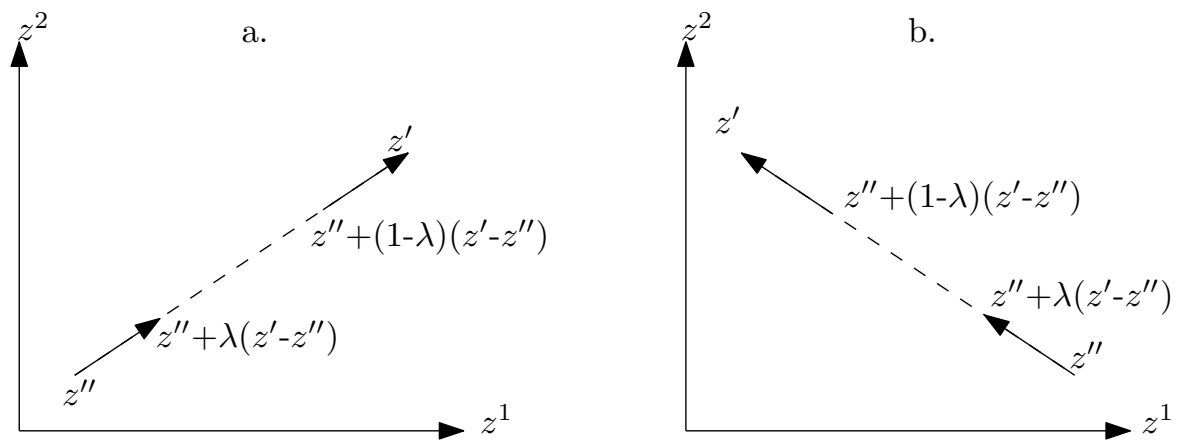

Figure 1: Geometrical intuition behind NII.

Proposition 1 Let $\succsim$ be a preference relation that admits an ordinal representation and assume further that it exhibits weak-monotonicity and strict convexity. Then for any finite set $Z \subset \mathbb{R}_{+}^{p}$, the restriction of $\succsim$ to $Z$ has a strictly concave representation.

This result shows that our approach is only slightly less general in its domain of application than the convex cones approach (which assumes ordinary rather than strict convexity). The need to work with a restriction of $\succsim$ to $Z$ does not seem a material limitation, as we are mainly interested in finding a representation over a (finite) reference set of points for which the DM has expressed preferences.

For the case where the DM is able to express preference intensities and this concept is taken as primitive, an alternative (and potentially easier) route into concavifiability than strict convexity is the following condition of nonincreasing preference intensities (inspired by a condition called "nonincreasing tradeoffs", introduced by Wakker 1988, p. 140 for the single-dimension case).

Definition 2 The relation $\succsim^{*}$ exhibits nonincreasing preference intensities (NII) if for all $z^{\prime}, z^{\prime \prime} \in Z$ and $\lambda \in(0,1),\left(z^{\prime \prime}+\lambda\left(z^{\prime}-z^{\prime \prime}\right) \leftarrow z^{\prime \prime}\right) \succsim^{*}\left(z^{\prime} \leftarrow z^{\prime \prime}+(1-\lambda)\left(z^{\prime}-z^{\prime \prime}\right)\right)$.

To get a sense of the meaning of the NII condition, consider how it might work in a biattribute environment. There are essentially two cases, shown in the panels a. and b. of Figure 1 . In a., when $\lambda<1 / 2$, monotonicity gives $z^{\prime} \succsim\left(z^{\prime \prime}+(1-\lambda)\left(z^{\prime}-z^{\prime \prime}\right)\right) \succsim\left(z^{\prime \prime}+\lambda\left(z^{\prime}-z^{\prime \prime}\right)\right) \succsim z^{\prime \prime}$. To satisfy NII, the DM must accept that she prefers the vector-increment $\lambda\left(z^{\prime}-z^{\prime \prime}\right)$ more intensely at $z^{\prime \prime}$ than at the more preferred point $z^{\prime \prime}+(1-\lambda)\left(z^{\prime}-z^{\prime \prime}\right)$. From this point of view, NII appears as a familiar diminishing marginal returns concept in a multiattribute environment. In b., point $z^{\prime \prime}$ can be seen to be high on $z^{1}$ and low on $z^{2}$ and for $z^{\prime}$ the situation is reversed. To satisfy NII, the DM must accept that however much she likes or dislikes the tradeoff $\lambda\left(z^{\prime}-z^{\prime \prime}\right)$ (a gain in $z^{2}$ at the expense of a loss in $z^{1}$ ), when it is offered at $z^{\prime \prime}$, she must like it less or dislike it more when it is offered at $z^{\prime \prime}+(1-\lambda)\left(z^{\prime}-z^{\prime \prime}\right)$. Thus NII can be seen as a condition on preference intensities for different tradeoffs along a ray in multiattribute space. These examples highlight the connection between NII and concavifiability (via diminishing marginal returns). This is formalized in Proposition 3 (all proofs are in appendix C).

Proposition 3 For weakly-monotonic preferences and preference intensities which are representable by a cardinal value function $u(\cdot)$, NII holds if and only if any representation of $\succsim^{*}$ is concave. 
In the preceding we assumed that the dimensions of $Z$ are continuous. Thus, the question arises as to how to deal with discrete attributes. We do not provide a full treatment for this case in this paper, but we do offer some ideas about how to proceed in theory and in practice. We note first that while this case is not a natural environment for the NII assumption, the same is true for preference-convexity (as $Z$ is not convex). To motivate the discussion, consider an example of a discrete scale, "Global Impact", used to evaluate research projects by a national funding body. Suppose the scale has three levels $\{0 ; 1 ; 2\}$ defined as follows: "No significance"; "National significance"; "International significance" (the scale is rather crude but it is sufficient for the point we wish to make). It is obviously meaningless to talk of decreasing marginal benefit over the interval $[0,2]$. Nevertheless, a DM may be asked to support (or refute) the assertion that, for any project, an improvement from 0 to 1 increases its value at least at much as one from 1 to 2. If so, we can construct a value function for "Global Impact" which is concave over the entire $[0,2]$ interval. Further, we can attempt to ensure concavifiability via the scale construction itself. For example, if the DM refutes the above assertion, we could replace the descriptor "National Significance" with the more stringent descriptor "National and European Significance" (say for a UK-based funding body), or, instead, introduce "European Significance" as a new intermediate level (so the scale would be $\{0 ; 1 ; 2 ; 3\}$ ). Then we would ask the DM again (and possibly repeat). In general, for a single attribute, the validity of the NII condition over a discrete numerical domain is sufficient to ensure concavifiability over a containing interval. We conjecture that this is also true for the multiple-attribute case (where some may be continuous), but we leave that open for further research. Finally, the problematic case could be avoided when it is possible to construct cardinal scales for individual attributes. For example, the worst and the best projects in terms of "Global Impact" could be assigned scores of 0 and 100; then every number within this interval is interpreted as a proportion of the benefit difference between these two projects (for that attribute). In such a case we obtain continuous scales and it is then appropriate to consider whether value exhibits diminishing marginal benefit over the product set of these attributes.

\section{Dominance and Efficiency}

In this section we deal with the problem of exploiting a DM's expressed ordinal preference and preference intensity statements over a set of alternatives. Denote by $R=\left\{z_{1}, \ldots, z_{n}\right\} \subseteq Z$, a finite reference set of alternatives where $z_{j}=\left(z_{j}^{1}, \ldots, z_{j}^{p}\right), \forall j \in J=\{1, \ldots, n\}$. Some of the alternatives could be hypothetical and only included to help elicit preferences. The set $R^{\prime}$ (with index set $J^{\prime}$ ) contains the 'real' alternatives and is a subset of $R$, a proper subset if there are hypothetical alternatives.

The general approach is standard in the literature. Let $\succsim_{R}^{*}$ be a relation representing a finite number of expressed preference intensity statements of the form:

$$
\left(z^{\prime} \leftarrow z^{\prime \prime}\right) \succsim_{R}^{*}\left(z^{\prime \prime \prime} \leftarrow z^{\prime \prime \prime \prime}\right) \quad z^{\prime}, z^{\prime \prime}, z^{\prime \prime \prime}, z^{\prime \prime \prime \prime} \in R,
$$

obtained from the DM. We include any possible expressed ordinal preference statements $z^{\prime} \succsim z^{\prime \prime}$ by allowing $z^{\prime \prime \prime}=z^{\prime \prime \prime \prime}$. Note that $\succsim_{R}^{*}$ will not be complete (if it is, then it must equal $\succsim^{*}$ and there is no need for further analysis).

Let $\mathcal{U}_{\mathcal{C N}}\left(\succsim_{R}^{*}\right)$ be the set of all nondecreasing concave functions over $Z$ that are consistent with $\succsim_{R}^{*}$, by which we mean that $u\left(z^{\prime}\right)-u\left(z^{\prime \prime}\right) \geq u\left(z^{\prime \prime \prime}\right)-u\left(z^{\prime \prime \prime \prime}\right)$, whenever one of the statements in (1) comprising $\succsim_{R}^{*}$ holds. To avoid triviality, we take $u(\mathbf{0})=0, u(\mathbf{1})=1$, for each $u(\cdot) \in \mathcal{U}_{\mathcal{C N}}\left(\succsim_{R}^{*}\right)$, where $\mathbf{0}$ and $\mathbf{1}$ are vectors or zeroes and ones. 


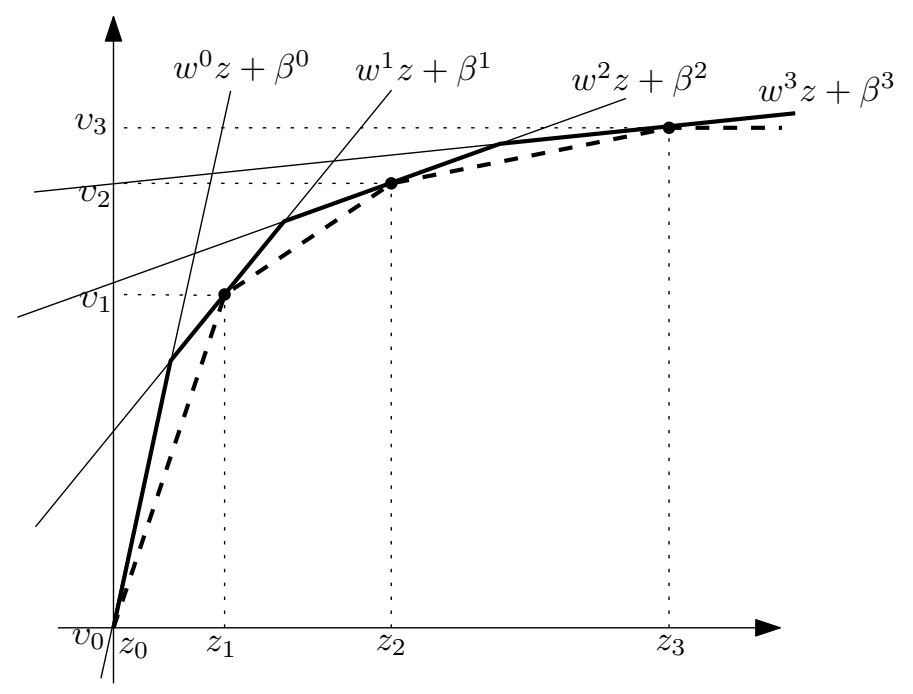

Figure 2: Graphical representation of CUT.

In general, for any collection $\mathcal{U}$ of value functions over $Z$, it is common in the literature to say that alternative $z^{\prime} \mathcal{U}$-dominates $z^{\prime \prime}$, if $u\left(z^{\prime}\right) \geq u\left(z^{\prime \prime}\right)$ for every $u(\cdot) \in \mathcal{U}$. Further, given a subset $R^{\prime}$ of alternatives from $R$, an alternative $z^{\prime} \in R^{\prime}$ is said to be $\mathcal{U}$-efficient in $R^{\prime}$ if there is some $u(\cdot) \in$ $\mathcal{U}$ for which $u\left(z^{\prime}\right) \geq u(z)$ for every $z \in R^{\prime}$. We shall be interested in characterizing efficiency and dominance when $\mathcal{U}=\mathcal{U}_{\mathcal{C N}}\left(\succsim_{R}^{*}\right)$. If $z^{\prime}$ is $\mathcal{U}_{\mathcal{C N}}\left(\succsim_{R}^{*}\right)$-efficient in $R^{\prime}$, then it is potentially a maximal element of $R^{\prime}$ with respect to the underlying preferences $\succsim$; otherwise it is certainly not maximal and can be discarded. Further, if $z^{\prime} \mathcal{U}_{\mathcal{C N}}\left(\succsim_{R}^{*}\right)$-dominates $z^{\prime \prime}$ then it follows that $z^{\prime} \succsim z^{\prime \prime}$.

The way in which we derive this characterization is introduced graphically in Figure 2. Consider four points: $z_{0}, z_{1}, z_{2}, z_{3}$. Each $z_{j}$ is mapped to a value score $v_{j}=w^{j} z_{j}+\beta^{j}$ which is the minimum over all mappings $w^{j} z_{k}+\beta^{j}$. In the multidimensional case the lines are hyperplanes and $w^{j}$ are $p$-vectors. Given this arrangement a concave nondecreasing value function that reproduces the value scores $v_{j}$ may be constructed as the pointwise minimum of a finite collection of affine functions (defined by the hyperplanes), as shown with the solid bold line. Alternatively, the boundary of the (extended) convex hull of the set of points $\left(v_{j}, z_{j}\right)$ can be used as the graph of a compatible value function, as indicated by the dashed bold line. The idea is that the set of all possible collections of hyperplanes which can be described in this way defines the set of all value assignments of interest.

In the general case, denote by $v=\left(v_{1}, \ldots, v_{n}\right)$ a vector of value assignments to the alternatives in $z_{1}, \ldots, z_{n}$, and let $\mathrm{Y}_{\mathcal{C N}}\left(\succsim_{R}^{*}\right)$ denote the set of those $v$ that are consistent with $\succsim_{R}^{*}$. Formally, define the polyhedron:

$$
\mathrm{Y}_{\mathcal{C N}}\left(\succsim_{R}^{*}\right)=\left\{v \in \mathbb{R}_{+}^{n} \mid \begin{array}{ll}
v_{j}-v_{k} \geq v_{l}-v_{m} & \text { if }\left(z_{j} \leftarrow z_{k}\right) \succsim_{R}^{*}\left(z_{l} \leftarrow z_{m}\right) \\
v_{j}=0, v_{k}=1 & \text { if } z_{j}=\mathbf{0}, z_{k}=\mathbf{1} \\
v_{j} \leq w^{k} z_{j}+\beta^{k} & \forall j, k \in J \text { with equality when } j=k \\
w^{j} \in \mathbb{R}_{+}^{p}, \beta^{j} \in \mathbb{R} & \forall j \in J
\end{array}\right\} .
$$


Here the first restriction guarantees consistency with $\succsim_{R}^{*}$; the third and fourth guarantee concavity. In what follows we will regularly suppress the arguments of $Y_{\mathcal{C N}}$. We note here that we have not explicitly considered the possibility of strict preference statements expressed by the DM to keep exposition simple. In fact, these can be included with little modification. As is commonplace in the literature, strict ordinal preferences, denoted $\succ_{R}$, can be included by introducing a small positive $+\varepsilon$ to the right-hand-sides of the corresponding constraints in (2). The value of $\varepsilon$ can be considered to be the minimum acceptable difference in any two value scores (given the scale normalization). Strict preference intensities can be handled in an analogous way. We now state the fundamental theorem.

Theorem 4 There is a one-to-one correspondence between value assignments $v \in \mathrm{Y}_{\mathcal{C N}}$ and the restrictions $\left.u\right|_{R}$ of functions $u(\cdot) \in \mathcal{U}_{\mathcal{C N}}\left(\succsim_{R}^{*}\right)$ to alternatives in $R$. For corresponding $v$ and $u(\cdot)$ we have $v_{j}=u\left(z_{j}\right)$ for all $z_{j} \in R$.

Theorem 4 makes it easy to characterize efficiency and dominance. For efficiency over a subset $R^{\prime} \subseteq R$, the following are equivalent:

a) Alternative $z_{j^{\prime}}$ is $\mathcal{U}_{\mathcal{C N}}\left(\succsim_{R}^{*}\right)$-efficient in $R^{\prime}$,

b) There is some $u \in \mathcal{U}_{\mathcal{C N}}\left(\succsim_{R}^{*}\right)$ such that $u\left(z_{j^{\prime}}\right) \geq u\left(z_{j}\right)$ for each $z_{j} \in R^{\prime}$,

c) There is some $v \in \mathrm{Y}_{\mathcal{C N}}\left(\succsim_{R}^{*}\right)$ such that $v_{j^{\prime}} \geq v_{j}$ for each $j \in J^{\prime}$, (invoking Theorem 4),

d) The set $\left\{v \in \mathrm{Y}_{\mathcal{C N}}\left(\succsim_{R}^{*}\right) \mid v_{j^{\prime}} \geq v_{j}\right.$ for each $\left.j \in J^{\prime}\right\}$ is nonempty.

For dominance, the following are equivalent:

e) $z_{j}$ is $\mathcal{U}_{\mathcal{C N}}\left(\succsim_{R}^{*}\right)$-dominates $z_{k}$,

f) $u\left(z_{j}\right) \geq u\left(z_{k}\right)$ for each $u \in \mathcal{U}_{\mathcal{C N}}\left(\succsim_{R}^{*}\right)$,

g) $v_{j} \geq v_{k}$ for each $v \in \mathrm{Y}_{\mathcal{C N}}\left(\succsim_{R}^{*}\right)$, (invoking Theorem 4),

h) $\min \left\{v_{j}-v_{k} \mid v \in \mathrm{Y}_{\mathcal{C N}}\left(\succsim_{R}^{*}\right)\right\} \geq 0$.

Of course, the conditions in statements d) and h) can each be checked by solving a linear program.

By Theorem 4, the feasibility of (2) is a necessary and sufficient condition for the representability of $\succsim_{R}^{*}$ by a concave and nondecreasing function. Kohlberg (1983) gives alternative conditions to ours in (2), but it is difficult to see how these can operationalize efficiency and dominance checks. For an alternative condition, effectively derived by "dualizing" (2), see Richter and Wong (2004).

\section{Interactive Procedure}

In this section we develop and interactive procedure for two types of multicriteria choice problems, namely: multicriteria discrete choice problems (i.e. problems of single choice from a set of 
explicitly listed alternatives) and 0-1 multicriteria optimization problems (i.e. optimization problems with only binary variables). The structure of the procedure is the same for both cases, so we introduce it using the generic problem of choosing from some feasible set of alternatives $X$, where each alternative in $X$ is scored across several criteria via criterion functions $f^{i}: X \rightarrow Z^{i}$. We then detail how to implement the procedure when $X$ takes the respective form for the two problems considered. For brevity, we use $F(x)=\left(f^{1}(x), \ldots, f^{p}(x)\right)$ and $F(X)=\{F(x) \mid x \in X\}$.

The general structure of the interactive procedure is standard. At every iteration $t$, the DM has expressed preferences denoted $\succsim_{t}^{*}$ over a set of alternatives $R_{t}$ (with index set $J_{t}$ ) and has identified an incumbent alternative $z_{t}^{i n c}=F\left(x_{t}^{i n c}\right)$ (for simplicity, we use $j^{\prime}$ to denote the index value of the incumbent alternative across all iterations). She is then asked to compare the incumbent to a challenger and declare her preference or indifference; then the cycle repeats. The critical idea is the way in which the challenger is generated: by identifying an alternative $z_{o}^{*} \in F(X)$ which maximizes the value difference with the incumbent across all value functions in $\mathcal{U}_{\mathcal{C N}}\left(\succsim_{t}^{*}\right)$. By invoking Theorem 4, this can be done by solving the following problem in (3), for which the optimal value is denoted by $A_{t}^{*}$ (we use asterisk-superscripts to denote optimal solutions in the ensuing).

$$
\max \left\{v_{o}-v_{j^{\prime}} \mid v \in \mathrm{Y}_{\mathcal{C N}}\left(\succsim_{t}^{*}\right), v_{o} \leq w^{j} z_{o}+\beta^{j} \forall j \in J_{t}, z_{o} \in F(X)\right\} .
$$

The solution of this problem is the only difference between the application of the procedure in the two problem types considered, and we describe this in the following two subsections. The full details for implementing the procedure outlined above are as follows. The reference set and the incumbent are initialized as $R_{1}=\{0,1\}$ and $z_{1}^{i n c}=\mathbf{1}$. As already remarked, the procedure continues by solving problem (3) at every iteration. At that point the optimal value $A_{t}^{*}$ is examined. If $A_{t}^{*}=0$ then the procedure terminates (there can be no alternative which would be strictly preferred to the incumbent). Otherwise we increment $t$, augment the reference set $R_{t}$ with the new challenger alternative $z_{0}^{*}$, ask the DM to compare the incumbent and challenger alternatives and state whether $z_{t-1}^{i n c} \succ z_{o}^{*}, z_{t-1}^{i n c} \sim z_{o}^{*}$ or $z_{o}^{*} \succ z_{t-1}^{i n c}$. Accordingly, we update $\succsim_{t}^{*}$ with the new preference statement (which translates to additional constraints on $\mathrm{Y}_{\mathcal{C N}}$ ). If the DM states that $z_{0}^{*} \succ z_{t-1}^{i n c}$, then we also update the information on the incumbent by setting $z_{t}^{i n c}=z_{0}^{*}$.

\subsection{The case of discrete choice problems}

In discrete choice problems alternatives are explicitly listed. Thus $X=F(X)$ and the constraints $z_{0} \in F(X)$ in (3) simply imply $z_{0} \in X=\left\{x_{1}, x_{2}, \ldots, x_{s}\right\}$. Fixing $z_{0}=x_{0}$ for some $x_{0} \in X$, the problem in (3) reduces to the following linear program in (4), which can be solved separately for all $x_{0} \in X$ :

$$
\max \left\{v_{o}-v_{j^{\prime}} \mid v \in \mathrm{Y}_{\mathcal{C N}}\left(\succsim_{t}^{*}\right), v_{o} \leq w^{j} x_{o}+\beta^{j} \forall j \in J_{t}\right\} .
$$

The alternative $x_{o}^{*}$ for which the value of problem (4) is maximal (with an arbitrary choice in case of ties) is the new challenger. In addition, alternatives for which the identified optimal value is nonpositive can be discarded (by Theorem 4, they are dominated by the incumbent). In section 5.4 we report on the use of this implementation in an application context. 


\subsection{The case of 0-1 multicriteria optimization problems}

For the case of 0-1 multicriteria optimization problems, the $f^{i}$ are linear: $f^{i}(x)=\sum_{q=1, \ldots, m} c_{q}^{i} x^{q}$, and $X$ is defined by linear inequalities: $X=\left\{x \in\{0,1\}^{m} \mid D x \leq b\right\}$. The second set of constraints in (3) can be written as: $\beta^{j}+w^{j} z_{o}=\beta^{j}+\sum_{q, i} c_{q}^{i} w_{i}^{j} x_{o}^{q}$. Each nonlinear term $w_{i}^{j} x_{o}^{q}$, the product of a binary and a continuous variable, can be linearized (details are in appendix A) and the problem converts to a standard mixed-integer program. In section 5.5 we report the computational results of this implementation for a set of 0-1 multicriteria optimization problems.

\subsection{Convergence}

We consider here whether the procedure based on solving problem (3) converges. Specifically, denote by $\dot{u}(\cdot)$ the "true" value function which represents $\succsim^{*}$ and by $\dot{u}^{\max }=\max _{z \in F(X)} \dot{u}(z)$ the "true" maximal attainable value. We consider whether $\dot{u}\left(z_{t}^{i n c}\right)$ converges to $\dot{u}^{\text {max }}$. For this we assume that the DM's responses are always consistent with ${ }^{*}$. In actual practice a DM is unlikely to be consistent in this way (see e.g. Korhonen, Moskowitz and Wallenius 1990), but that should not stop us exploring such a scenario from a theoretic point of view. We report on how we dealt with inconsistent responses in practice in sub-section 5.4.

We show that the procedure, which we label CUT-interactive, converges. Further, this is true not only for the two problem types of problems considered above, but for a very general class of problems. Of course, in the general case the problem in (3) is nonlinear, even when the description of $F(X)$ is not. Thus, in cases where $F(X)$ is linear and for which any existing approaches do not rely on nonlinear problems, the procedure would not be a contender. Nevertheless, these would be special cases to which our result applies. Further, the result would also apply to nonlinear multicriteria problems (see e.g. Miettinen 1999). The general class of problems is defined as follows. We assume that $X$ and $F(X)$ are non-empty and compact and that $Z=\mathbb{R}_{+}^{p}$. We also assume that there exists a positive $r$ so that $z \geq r \mathbf{1}, \forall z \in F(X)$. We underline that we make no assumption regarding convexity for $X$ and $F(X)$.

Theorem 5 In the procedure CUT-interactive, $\dot{u}\left(z_{t}^{\text {inc }}\right)$ converges to $\dot{u}^{\max }$ as $t \rightarrow \infty$.

\subsection{User Tests}

We conducted a series of user tests based on a flat-choice problem with seven subjects, who were all professional analysts. We framed the problem as choosing a flat in Paris to stay for six months, from a list of 100 flats based on data from a flat-rental website (33 flats were dominated, but were kept in for the benefit of detecting satiation). Flats were characterized by number of bedrooms, average size of bedrooms, distance from place of work (operationalized as estimated leisure time after work and commute) and cost (operationalized as monthly net income). To create a value scale, we used two artificial flats: the ideal and the nadir, constructed using the maximum/minimum value across all flats in each criterion, and assigned value scores of 0 and 100.

At each iteration, we showed users a new flat (challenger), asked them to compare to the best flat they had seen so far (incumbent) and declare their preference (or indifference). Their responses generated preference constraints as in (2) (but also adding an $\varepsilon=0.01$ to the right-hand-sides for the case of strict preferences). We also asked them whether the intensity of their preference 
was "weak", "moderate" or "strong". These responses generated weak preference-intensity constraints as in (2). For example, if a subject declared "moderate" preference for $z_{a}$ over $z_{b}$ and "strong" preference for $z_{c}$ over $z_{d}$, the constraint was: $v_{c}-v_{d} \geq v_{a}-v_{b}$. Optionally, subjects were asked to rank the challenger amongst previously ranked alternatives. The questioning was followed by a brief pause (a few seconds) for computations, to conduct a set of inconsistency checks, discard dominated alternatives and compute a new challenger (as discussed in Section 5.1). We then shared with users the computed maximum value difference between unseen alternatives and the incumbent and asked them if they wanted to continue, in which case the process would repeat. Where judgements exhibited inconsistency, we shared the reason for the inconsistency with users and asked them whether they wanted to revise (or even discard) any of their previous judgments, or terminate the procedure. In appendix B we describe the inconsistency checks and provide details of the iteration-by-iteration evolution of the procedure for a representative subject.

We emphasized to subjects that they could stop at any time and for any reason, including boredom or frustration. All continued to the point where a most preferred alternative was demonstrably reached, i.e. when the maximum value difference between unseen alternatives and the incumbent reached 0 (convergence). The procedure was experienced as progressive: later choices were experienced as being more difficult because search had narrowed to a set of reasonably good alternatives ("you can see that the choices are getting reduced ... and that the choices converge to something."). The number of iterations ranged from 13 to 28 (averaging 21) for completion and from 3 to 16 (averaging 10) for reaching the most preferred alternative. Completion times were between 25 minutes and an hour, with an average of 43 minutes.

Subjects always responded to the choice and preference intensity questions. They found it most natural to rank objects that fell within the top five of those already seen, as their interest focused on the most attractive options. Four subjects reported finding the intensity of preference question harder and the other three found ranking harder. Several subjects remarked that a visual scale for the alternatives would have made it easier to answer these questions.

Responses exhibited between 1 and 5 inconsistencies, averaging 2 per subject. Predominantly, these took the form of a contradiction between ranking and intensity judgements: e.g. subjects might indicate that they preferred $a$ to $b$ strongly and $a$ to $c$ weakly, but also preferred $a$ to $b$ to $c$. On all occasions, subjects chose to revise their intensity judgements accordingly. Two subjects gave responses that directly contradicted the concavifiability of the combined ranking and intensity judgments; this occurred twice for one of the subjects and once for the other. On these occasions we discussed with them the normative implications of the NII assumption and asked them whether they wanted to proceed. Both subjects chose to discard their latest ranking response, as they were not bothered about the rank of a non-preferred alternative. The former of these two users pointed out, after completion, a possible explanation for the inconsistencies: he was using a cut-off value for the number of rooms. In practice this situation can by avoided by pre-filtering the alternatives (e.g. as in all web-based tools for flat search). No other types of inconsistency were observed, i.e. ranking and intensity judgments were always internally consistent with concavifiability.

The degree of enthusiasm for the procedure varied from highly enthusiastic ("this is a lot more intuitive" than scoring and weighting) to cautious interest (one multicriteria practitioner accepted that DMs could holistically compare 4 attributes, but doubted their ability to handle substantially greater numbers). All subjects could imagine using the procedure for a real decision. Indeed, one subject commented "last year I bought a flat, this is much easier [than unsupported choice]". 


\subsection{Computational Tests}

To explore the computational behavior of the interactive procedure for $0-1$ multicriteria optimization problems, we applied it to a project-portfolio selection (knapsack) problem. Computations were performed on a PC with an Intel E8300 $2.83 \mathrm{GHz}$ processor and 3.25GB of memory, using the CPLEX 12.4 mixed-integer programming solver. Results were validated by generating all nondominated solutions using a version of the algorithm by Captivo et al. (2003).

The problem data (provided to us by Larry Phillips) come from a decision analysis at a hightechnology company that wanted to construct an R\&D portfolio from a set of twenty-one projects. Projects are evaluated on three criteria: "Revenue", "Innovativeness" and "Strategic-fit". The individual criterion functions are assumed additive over projects (i.e. for each criterion the aggregate portfolio score is the sum of the scores of the constituent projects). The feasible set of portfolios consists of those that fall within budget. We considered three budget levels, corresponding to $25 \%, 50 \%$ and $75 \%$ of the total cost of all available projects. For the purpose of simulating the DM we considered two value functions: $U^{1}(z)=\min _{i}\left\{f^{i}(x)\right\}$ and $U^{2}(z)=\prod_{i} f^{i}(x)$. Function $U^{1}$ is concave. The preferences it represents are in fundamental disagreement with the preferential independence conditions underlying additive-type value models. Function $U^{2}$ is merely quasiconcave. It was specifically chosen to illustrate that the interactive procedure may also be used in the more "standard" setting of quasi-concave-representable preferences with no use of preference intensity information, for problems with finite sets of alternatives (which can be, as in this case, explicitly unknown and very large).

We examined several combinations of different types of preference information. In the most basic scenario, labeled $b$, the DM is only asked to compare the challenger and incumbent at every iteration and declare preference or indifference. In scenario $r$, the DM is asked to maintain a ranking of alternatives encountered across iterations. This may require many comparisons, so we also considered scenario $r 5$, where the DM only maintains a ranking of the top five alternatives she has seen so far (i.e. she disregards any challengers that do not make it into the top five). To introduce preference intensities, we used scenario $b-c l$, where the DM compares the challenger to the incumbent and declares weak/moderate/strong preference or indifference (this produces three clusters of challenger-incumbent pairs which then define constraints as in (2)). In view of our experience with the user tests, we used scenario $r 5-c l 5$, to specifically consider situations where the DM's preference intensity perception may vary across pairs of alternatives. We constructed this by using the top-five ranking of scenario $r 5$ and grouping the four pairs of successively ranked alternatives into the three preference intensity classes (weak/moderate/strong preference).

In Table 1 we report the number of iterations and computer runtime (in seconds), as well as the number of binary comparisons. Note that the scenarios including preference intensities also require one qualitative intensity judgment per iteration. Results are reported for three events: a) "Solution" (Sol.), the point at which a most preferred solution/alternative is discovered (one which maximizes the true value function), even if this is not verifiable at that stage; b) "Satisfaction" (Sat.), the point at which the value of optimization problem (3) falls below 10, indicating that at worst the current incumbent cannot be more than 10 "vals" (units of value) worse than the true most preferred point (a val is normalized as the value difference between the zero vector and unit vector); c) "Completion" (Comp.), the point at which convergence to a most preferred alternative has been verified. We emphasize the ability to provide information on "Satisfaction" as a particular strength of CUT. This gives the DM a sense of the greatest possible value difference from the most preferred point, and hence the opportunity cost of abandoning search. In all reported cases the 
Table 1: Performance of the interactive procedure for all scenarios.

\begin{tabular}{|c|c|c|c|c|c|c|c|c|c|c|c|}
\hline \multirow[t]{2}{*}{$U$} & \multirow[t]{2}{*}{ Budget } & \multirow[t]{2}{*}{ Scenario } & \multicolumn{3}{|c|}{ Iterations } & \multicolumn{3}{|c|}{ Runtime (sec.) } & \multicolumn{3}{|c|}{ Comparisons } \\
\hline & & & Sol. & Sat. & Compl. & Sol. & Sat. & Compl. & Sol. & Sat. & Compl. \\
\hline \multirow{5}{*}{$U^{1}$} & \multirow{5}{*}{$25 \%$} & $b$ & 5 & 9 & 15 & 2.82 & 11.53 & 63.46 & 4 & 7 & 14 \\
\hline & & $r$ & 5 & 9 & 13 & 1.93 & 9.75 & 41.20 & 7 & 13 & 34 \\
\hline & & $r 5$ & 5 & 9 & 13 & 2.25 & 10.23 & 41.20 & 7 & 14 & 26 \\
\hline & & $b-c l$ & 5 & 9 & 10 & 2.59 & 10.80 & 14.60 & 4 & 7 & 9 \\
\hline & & $r 5-c l 5$ & 5 & 9 & 10 & 2.03 & 10.69 & 13.85 & 7 & 13 & 17 \\
\hline \multirow{5}{*}{$U^{1}$} & \multirow{5}{*}{$50 \%$} & $b$ & 9 & 10 & 42 & 13.74 & 19.00 & 2813.70 & 8 & 8 & 41 \\
\hline & & $r$ & 9 & 11 & 21 & 12.00 & 22.71 & 147.25 & 16 & 17 & 66 \\
\hline & & $r 5$ & 9 & 11 & 23 & 11.92 & 21.36 & 217.92 & 16 & 17 & 57 \\
\hline & & $b-c l$ & 9 & 10 & 24 & 13.98 & 19.22 & 423.48 & 8 & 8 & 23 \\
\hline & & $r 5-c 15$ & 8 & 9 & 16 & 9.14 & 13.87 & 75.27 & 10 & 10 & 36 \\
\hline \multirow{5}{*}{$U^{1}$} & \multirow{5}{*}{$75 \%$} & $b$ & 6 & 9 & 16 & 1.65 & 3.81 & 27.55 & 5 & 7 & 15 \\
\hline & & $r$ & 6 & 9 & 14 & 1.36 & 3.84 & 15.83 & 8 & 13 & 34 \\
\hline & & $r 5$ & 6 & 9 & 14 & 1.26 & 3.99 & 16.26 & 8 & 13 & 30 \\
\hline & & $b-c l$ & 6 & 9 & 13 & 1.73 & 3.03 & 12.26 & 5 & 7 & 12 \\
\hline & & $r 5-c l 5$ & 6 & 9 & 13 & 1.9 & 4.57 & 13.17 & 8 & 13 & 29 \\
\hline \multirow{3}{*}{$U^{2}$} & \multirow{3}{*}{$25 \%$} & $b$ & 1 & 15 & 25 & 0.12 & 45.32 & 255.61 & 15 & 13 & 24 \\
\hline & & $r$ & 1 & 10 & 19 & 0.12 & 15.71 & 116.83 & 10 & 17 & 82 \\
\hline & & $r 5$ & 1 & 10 & 19 & 0.12 & 16.13 & 124.40 & 10 & 17 & 46 \\
\hline \multirow{3}{*}{$U^{2}$} & \multirow{3}{*}{$50 \%$} & $b$ & 6 & 15 & 51 & 4.49 & 82.23 & 6446.46 & 5 & 13 & 50 \\
\hline & & $r$ & 4 & 13 & 25 & 1.50 & 49.48 & 455.37 & 3 & 30 & 131 \\
\hline & & $r 5$ & 6 & 13 & 28 & 4.66 & 52.49 & 715.78 & 7 & 24 & 65 \\
\hline \multirow{3}{*}{$U^{2}$} & \multirow{3}{*}{$75 \%$} & $b$ & 13 & 13 & 26 & 14.99 & 14.99 & 183.60 & 12 & 11 & 25 \\
\hline & & $r$ & 9 & 11 & 17 & 5.69 & 8.28 & 38.74 & 17 & 18 & 83 \\
\hline & & $r 5$ & 9 & 11 & 17 & 6.46 & 9.52 & 33.70 & 17 & 18 & 84 \\
\hline
\end{tabular}

10-val "Satisfaction" clause corresponded to less than 5\% of the value of the "true" optimum.

Since we have identified the ability to inform the DM about "Satisfaction" and the capacity to utilize preference intensities as two distinctive features of CUT relative to existing approaches, a particularly interesting question is whether these prove useful in improving the overall effectiveness of the interactive procedure. The results reported here indicate that this is indeed the case.

In practice, DMs may be unwilling to engage with interactive procedures for an extended period of time or many iterations, especially when they have no sense of how much better they could do by continuing. Thus, reaching a good solution fast would be instrumental to the applicability of the procedure. These results indicate that the interactive procedure performs very well on that account. For all scenarios, the procedure is very fast in reaching the most preferred alternative ("Solution"). Perhaps more importantly, the procedure is also very fast in reaching "Satisfaction", where acceptable assurances can be given to the DM about the opportunity cost of premature termination. In fact, for all these cases the DM would never do better by continuing past "Satisfaction", i.e. the incumbent at "Satisfaction" was always the "true" optimum.

The use of preference-intensity information provides significant performance improvements; 
and this is an important advantage of CUT compared to approaches which can only utilize ordinal information. For the problems where the "standard" scenario $b$ is performing adequately, the improvement gained from including preference intensities (or rankings) may be outweighed by the increased judgemental burden to the DM. Nevertheless, the usefulness of preference intensities clearly comes out in the "difficult" problems: for example, compared to $b$ and $r 5$ under $U^{1}$ and a $50 \%$ budget level, scenarios $b-c l$ and $r 5-c l 5$ respectively achieve reductions in time for "Completion" by factors of more than six and almost three. The improvements can be seen across the three reported events. It is particularly appealing that in all cases much of the performance gains can still be achieved when reducing the judgemental load: by asking the DM for top-five rankings only and without insisting that her intensity of preference responses are reliable when comparing alternatives outside the top-five ranking. These results are in agreement with our experience in the user tests, in indicating that preference scenario $r 5-c l 5$ provides a very attractive balance between computational efficiency and judgemental burden to the DM.

\section{Conclusion}

This paper presented a way to handle a DM's expressed (concavifiable) preferences of wide and general applicability. We call this approach CUT. We have shown how CUT can be used to verify dominance relations on a set of discrete alternatives. We introduced an interactive implementation of CUT and showed that it does (from a theoretic point of view) converge. The practicality of the approach was illustrated in a series of user tests for a flat-choice problem and a set of computational tests for a portfolio decision analysis problem.

The methods and ideas we presented raise several opportunities for practice and for research. In practice, the additive value model is widely used to support decision making and implemented in several user-friendly software packages. Often, these allow users to input ranges for the weights and value scores, or qualitative judgments of relative magnitude, and make various extended dominance checks. The tools developed here allow for computing similar dominance checks, which exchange the additivity assumption for concavity. Regarding the application of procedure in Section 5 to other problem types, an interesting question for research is whether an alternative way of generating challengers can be found that preserves the procedure's convergence properties. From a practical point of view, DMs may benefit more from a way to systematically explore feasible alternatives. To that end it would be interesting to consider the use of different types of preference information and alternative interactive designs: e.g. examine if it is possible to design a procedure that only requires tradeoffs between two attributes (and whether it can converge), or even examine the use of DM-specified local trade-offs (i.e. elicit values for different $w^{j}$ in (2)). Finally, another interesting question is how to use CUT for problems of multicriteria choice under uncertainty. The interpretation of concavity in this setting (risk-aversion) and the "natural" cardinality of the environment, suggest that this would be a promising direction of research.

\section{References}

Angillela, S., S. Greco, and B. Matarazzo. 2010. Non-additive robust ordinal regression: A multiple criteria decision model based on the Choquet integral. European Journal of Operational Research 2010(1) 277-288. 
Belton, V. and T. J. Stewart. 2002. Multiple Criteria Decision Analysis: An Integrated Approach. Kluwer, Boston.

Captivo, M. E., J. Clímaco, J. Figueira, E. Martins and J. L. Santos. 2003. Solving bicriteria 0-1 knapsack problems using a labelling algorithm. Computers and Operations Research 30 1865-1886.

de Finetti, B. 1949. Sulle stratificazioni convesse. Annali di Matematica Pura ed Applicata 30(4) 173183.

Dyer, J. and R. A. Sarin. 1979. Measurable Multiattribute Value Functions. Operations Research 22 810-822.

Dyer, J. MAUT - Multiattribute Utility Theory. 2005. Figueira, J. R., S. Greco and M. Ehrgott, eds. Multiple Criteria Decision Analysis: State of the Art Surveys. Springer, NY.

Farquhar, P., and L.R Keller. 1989. Preference intensity measurement. Annals of Operations Research 19(1) 205-217.

Fenchel, W. 1955. Über konvexe funktionen mit vorgeschriebenen niveaumannigfaltigkeiten. Mathematische Zeitschrift 63(1) 496-506.

Fishburn P. C. and P. Wakker. 1995. The Invention of the Independent Condition for Preferences. Management Science 41(7) 1130-1144.

Geoffrion, A., J. S. Dyer, and A. Feinberg. 1972. Interactive Approach for Multi-Criterion Optimization, with an Application to Operation of an Academic Department. Management Science 19(4) 357-368.

Greco, S., R. Słowiński, J. R. Figueira, V. Mousseau. 2010. Robust ordinal regression. M.Ehrgott, J.Figueira, S.Greco eds. Trends in Multiple Criteria Decision Analysis. Springer, NY.

Greco, S., B. Matarazzo, and R. Słowiński. 2001. Rough sets theory for multicriteria decision analysis. European Journal of Operational Research 129(1) 1-47.

Hazen, G. B. 1983. Preference Convex Unanimity in Multiple Criteria Decision-Making. Mathematics of Operations Research 8(4) 505-516.

Jacquet-Lagrèze, E. and J. Siskos. 1982. Assessing a set of additive utility functions for multicriteria decision-making: the UTA method. European Journal of Operational Research 10(2) 151-184.

Jacquet-Lagèze, E., R. Meziani and R. Słowiński. 1987. MOLP with an Interactive Assessment of a Piecewise Linear Utility Function. European Journal of Operational Research 31(3) 350-357.

Kalandrakis, T. 2010. Rationalizable voting. Theoretical Economics 5(1) 93-125.

Karsu, Ö, A. Morton, and N. Argyris. 2012. Incorporating Preference Information in Multicriteria Problems with Equity Concerns. Working Paper, Management Science Group, Department of Management, London School of Economics and Political Science, London.

Keeney, R. and H. Raiffa. 1993. Decisions with Multiple Objectives: Preferences and Value Tradeoffs. Cambridge University Press, NY 
Keeney, R. L. and D. von Winterfeldt. 2007. Practical Value Models. W. Edwards, R.F. Miles and D. von Winterfeldt, eds. Advances in Decision Analysis. Cambridge, CUP.

Köbberling, V. 2006. Strength of preference and cardinal utility. Economic Theory 27(2) 375-391.

Kohlberg, E. 1983. Consistency of finite systems of convex inequalities. Mathematical Programming 25 359-362.

Korhonen, P., J. Wallenius and S. Zionts. 1984. Solving the Multiple Criteria Problem using Convex Cones. Management Science 30(11) 1336-1345.

Korhonen, P., H. Moskowitz and J. Wallenius. 1986. A Progressive Algorithm for Modeling and Solving Multiple-Criteria Decision Problems. Operations Research 34(5) 726-731.

Korhonen, P., H. Moskowitz and J. Wallenius. 1990. Choice behavior in interactive multiplecriteria decision making. Annals of Operations Research 23(1) 161-179.

Korhonen, P., H. Moskowitz, P. Salminen and J. Wallenius. 1993. Further developments and tests of a progressive algorithm for multiple criteria decision making. Operations Research 41(6) 10331045.

Krantz, D. H., R. D. Luce, P. Suppes and A. Tversky. 1971. Foundations of Measurement, Vol. 1. Academic Press, NY.

Miettinen, K. 1999. Nonlinear Multiobjective Optimization. Kluwer, Boston.

Phillips, L. D. 1984. A theory of requisite decision models. Acta Psychologica 56 29-48.

Prasad, S., M.H. Karwan and S. Zionts. 1997. Use of Convex Cones in Interactive Multiple Objective Decision Making. Management Science 43(5) 723-734.

Ramesh, R., M. H. Karwan and S. Zionts. 1989. Preference Structure Representation Using Convex Cones in Multicriteria Integer Programming. Management Science 35(9) 1092-1105.

Richter, M. and K. Wong. 2004. Concave utility on finite sets. Journal of Economic Theory 115 341357.

Roy, A., P. Mackin, J. Wallenius, J. Corner, M. Keith, G. Schymik and H. Arora, H. 2008. An interactive search method based on user preferences. Decision Analysis 5(4) 203-229.

Roy, B. 1993. Decision science or decision-aid science?. European Journal of Operational Research 66 184-203.

Salo, A. and R. P. Hämäläinen. 2010. Preference Programming - Multicriteria Weighting Models under Incomplete Information. C. Zopounidis and P. M. Pardalos, eds. Handbook of Multicriteria Analysis. Springer, NY.

Shin, W. S. and A. Ravindran. 1991. Interactive multiple objective optimization: Survey I: continuous case. Computers \& Operations Research 18 97-114.

Siskos, Y., E. Grigoroudis and N. F. Matsatsinis. 2005. UTA Methods. J. Figueira, S. Greco and M. Ehrgott, eds. Multiple Criteria Decision Analysis: State of the Art Surveys. Springer, NY. 
Stewart, T. J. 1987. Pruning of decision alternatives in multiple criteria decision-making based on the UTA method for estimating utilities. European Journal of Operational Research 28(1) 79-88.

Topkis, D. M. 1998. Supermodularity and Complementarity. Princeton University Press, Princeton, NJ.

Vanderpooten, D. 1989. The interactive approach in MCDA: a technical framework and some basic conceptions. Mathematical and Computer Modelling 12 1213-1220.

von Winterfeldt, D. and W. Edwards. 1986. Decision Analysis and Behavioral Research. Cambridge University Press, NY.

Wakker, P. 1988. Additive Representations of Preferences: A New Foundation of Decision Analysis. Kluwer, Dordrecht.

Zionts, S. and J. Wallenius 1983. An Interactive Multiple Objective Linear-Programming Method for a Class of Underlying Non-Linear Utility-Functions. Management Science 29(5) 519-529.

\section{A Details about the linearization of Problem (3)}

We detail here how the to linearize problem (3) for the 0-1 multicriteria optimization case. As observed in section 5.2, the nonlinearity is limited to the quadratic terms $w_{i}^{j} x^{q}$, where $w_{i}^{j} \geq 0$ and $x^{q} \in\{0,1\} \forall(i, j, q)$ (note we have dropped the subscript $o$ for simplicity - it was only used to refer to a specific $x_{0} \in X$ ). These can be linearized by applying a well known method (see e.g. Glover 1975). Specifically, each term is linearized by the introduction of new variables $y_{i}^{j q} \geq$ 0 , and three linear constraints for each of these variables which collectively ensure that $y_{i}^{j q}=$ $w_{i}^{j} x^{q} \forall(i, j, q)$. Then we may add these constraints to problem (3) and replace all terms $w_{i}^{j} x^{q}$ with $y_{i}^{j q}$, thus transforming it into an mixed-integer program. The constraints to be added for each term $w_{i}^{j} x^{q}$ are as follows: $w_{i}^{j}-\left(1-x^{q}\right) \leq y_{i}^{j q} \leq w_{i}^{j}+\left(1-x^{q}\right)$, and $y_{i}^{j q} \leq M x^{q}$, where $M$ is large enough to be a valid upper bound for all $w_{i}^{j}$. It is easy to see that these constraints ensure that $y_{i}^{j q}=w_{i}^{j}$ when $x^{q}=1$ and $y_{i}^{j q}=0$ when $x^{q}=0$, i.e. that $y_{i}^{j q}=w_{i}^{j} x^{q}$ as required. For computational efficiency, the "big M's" in the constraints can be set to individual values. We describe how to derive bounds $M_{j} \forall j$. First, observe that for $j^{*}: z_{j^{*}}=\mathbf{1}$ the equality constraint in (2) (also in (3)) requires $w^{j^{*}} \mathbf{1}+\beta^{j^{*}}=1$, therefore $w_{i}^{j^{*}} \leq 1 \forall i$ and $\beta^{j^{*}} \leq 1$ (recall $w_{i}^{j^{*}} \geq \mathbf{0}$ and $\beta^{j^{*}} \geq 0$ ), so we may set $M_{j^{*}}=1$. Then for $j: z_{j} \neq \mathbf{0}, z_{j} \neq \mathbf{1}$, we get (from (2)): $w^{j}\left(\min _{i}\left\{z_{i}^{j}\right\}\right) \mathbf{1} \leq$ $\left.w^{j} z_{j} \leq w^{j} z_{j}+\beta^{j}=v_{j} \leq w^{j^{*}} z_{j}+\beta^{j^{*}} \leq \mathbf{1} z_{j}+1 \Rightarrow w_{i}^{j} \leq\left(\mathbf{1} z_{j}+1\right) /\left(\min _{i}\left\{z_{i}^{j}\right\}\right)\right)$, so we may set $\left.M_{j}=\left(\mathbf{1} z_{j}+1\right) /\left(\min _{i}\left\{z_{i}^{j}\right\}\right)\right) \forall j: z_{j} \neq \mathbf{0}, z_{j} \neq \mathbf{1}$. Finally, for $j^{\prime}: z_{j^{\prime}}=\mathbf{0}$, observe that (from (2)) $\beta^{j^{\prime}}=0$ and any $w^{j^{\prime}}$ satisfies $w^{j^{\prime}} \mathbf{0}=v_{j}^{\prime}=0$. Thus we only need a bound $M_{j^{\prime}}$ sufficiently high to guarantee collective feasibility for the inequalities $v_{j} \leq w^{j^{\prime}} z_{j}+\beta^{j^{\prime}} \forall j: z_{j} \neq \mathbf{0}$. It is easy to verify that the maximum of all the other $M_{j}$ values is such a bound. 


\section{B Details about the user tests}

To conduct the user tests, we recruited a convenience sample of seven subjects. All were professional analysts: four consultants, an operations research analyst in the UK Government, a retired former analyst in a UK Government agency and an Audit Manager at the UK's National Audit Office. We were keen to recruit subjects with some exposure to or experience of multicriteria methods who would be able to make an informed comparison of the questioning protocol with the traditional elicitation of scores and weights. We deliberately avoided subjects from academia, as the aim was to get an understanding of practical usability rather than methodological innovativeness. We made appointments with subjects for an hour and a half, to allow for an introduction to the task, use of the interactive procedure, and debrief afterwards. To pinpoint inconsistencies, a series of linear programs (feasibility systems) were solved. We first checked transitivity for the combined responses from incumbent-challenger comparisons and ranking questions. A further transitivity check was performed by adding the preference intensities. We then conducted four further checks for the concavifiability of the elicited preferences: first by using the challengerincumbent preferences only, then separately adding rankings or intensity judgements and finally using all preference information. In Table 2 we provide the iteration-by-iteration evolution of the procedure for a representative subject. The rows of the table show the new alternatives generated at each iteration. The process begins by showing to the subject the ideal and the nadir, in order to give a sense of the spread of the data - clearly the ideal point should be strongly preferred to the nadir. Then alternative 3 is generated and compared to the nadir at which point it becomes the incumbent, but is rapidly replaced in turn by 4 . After a couple of iterations, alternative 7 becomes the incumbent, but then the subject expresses the view that 8 is strongly preferred to 7 , and so 8 becomes the incumbent. The judgment of strong preference proves to be problematic, however, as 9 is judged to be moderately less preferred to 8 but is ranked lower than 7 . This provokes an inconsistency and the subject revises his earlier judgment of the strength of preference between 8 and 7 as moderate. A very similar problem is encountered on the next iteration, and the subject revises again the strength of preference judgment between 8 and 7 as weak. After this there are no further inconsistencies and the procedure terminates after a further 8 iterations. It became apparent on discussion with the subject that the reason for the inconsistencies was an ambiguity in the concept of "strength of preference": he had interpreted a strong preference as a "definite preference". Alternative 7 is in fact dominated by 8: for him it was thus "definitely better" (even though it was not better by much), and thus he declared a strong preference. Upon clarification this was revised, initially to moderate and then to weak, and the inconsistencies were resolved. Obviously neither of these inconsistencies would have occurred had we enforced the use of a strict monotonicity framework and/or removed the dominated alternatives, as may be reasonably done in practice. Nevertheless, their occurrence does highlight the importance of clearly communicating what is meant by "strong" or "weak" preference in this context. It also highlights that this interactive process is a learning process. Alerting the DM to such inconsistencies (instead of resolving them by e.g. discarding some preferences statements) provides further opportunity for the DM to deliberate and reflect on their preferences.

\section{Proofs}

In addition to the full proofs, we also provide short sketches of the proof strategies. 
Table 2: Iterations of the procedure for a representative subject.

\begin{tabular}{|c|c|c|c|c|c|}
\hline Altern. & Bedr. & $\begin{array}{l}\text { Av. } \\
\text { Area } \\
\text { (sq m) }\end{array}$ & $\begin{array}{l}\text { Leisure } \\
\text { time } \\
\text { (hours) }\end{array}$ & $\begin{array}{l}\text { Monthly } \\
\text { income } \\
(£)\end{array}$ & Decision maker judgment \\
\hline 1 (nadir) & 1 & 4 & 3 & 320 & \\
\hline 2 (ideal) & 5 & 45 & 6 & 2080 & (Strongly preferred to nadir) \\
\hline 3 & 1 & 27 & 4.5 & 1776 & Strongly preferred to nadir - becomes incumbent \\
\hline 4 & 2 & 22 & 4.5 & 1640 & Weakly preferred to 3 - becomes incumbent \\
\hline 5 & 5 & 5 & 4.5 & 640 & Strongly less preferred to 4 ranked as 3 rd, behind 4 and 3 \\
\hline 6 & 4 & 7 & 6 & 600 & Strongly less preferred to 4 ranked as 3 rd, behind 4,3 \\
\hline 7 & 2 & 11 & 6 & 1360 & Weakly preferred to 4 - becomes incumbent \\
\hline 8 & 2 & 18 & 6 & 1440 & Strongly preferred to 7 - becomes incumbent \\
\hline 9 & 3 & 10 & 5.5 & 1520 & Moderately less preferred to 8 ranked as 5 th, behind $8,7,4,3$ \\
\hline \multicolumn{6}{|c|}{$\begin{array}{l}\text { Inconsistency detected: } 8 \text { strongly preferred to } 7,8 \text { moderately preferred to } 9 \text {, but } 9 \text { is ranked lower than } 7 \text {. } \\
\text { Subject reviews judgements and concludes that } 8 \text { should be moderately preferred to } 7 \text {. }\end{array}$} \\
\hline 10 & 1 & 35 & 6 & 1880 & Weakly less preferred to 8 ranked as $3 \mathrm{rd}$, behind 8 and 7 \\
\hline \multicolumn{6}{|c|}{$\begin{array}{l}\text { Inconsistency detected: } 8 \text { weakly preferred to } 10,8 \text { moderately preferred to } 7 \text {, yet } 10 \text { is ranked lower than } 7 \text {. } \\
\text { Subject reviews judgements and concludes that } 8 \text { should be weakly preferred to } 7 \text {. }\end{array}$} \\
\hline 11 & 2 & 13 & 6 & 1800 & Moderately preferred to 8 - becomes incumbent \\
\hline 12 & 2 & 17 & 5.5 & 1752 & Weakly less preferred to 11 ranked as 2 nd \\
\hline 13 & 3 & 12 & 5.5 & 1200 & Moderately less preferred to 11 not ranked \\
\hline 14 & 2 & 17 & 6 & 1640 & Weakly less preferred to 11 ranked as 3rd, behind 11 and 12 \\
\hline 15 & 2 & 13 & 5 & 1960 & Weakly preferred to 11 - becomes incumbent \\
\hline 16 & 1 & 45 & 5 & 1774 & Moderately less preferred to 15 ranked as 4 th, behind $15,11,12$ \\
\hline 17 & 2 & 16 & 5 & 1856 & Moderately less preferred to 15 ranked as 3 rd, behind 15,11 \\
\hline 18 & 2 & 12 & 6 & 1856 & Weakly preferred to 15 - becomes incumbent \\
\hline
\end{tabular}




\section{C.1 Proposition 3}

The necessary part of the Proposition is a relatively straightforward consequence of the definition of concavity. The idea behind the proof of the sufficient part is more complicated. We have to show that if NII holds, then $u\left(\lambda z^{\prime}+(1-\lambda) z^{\prime \prime}\right) \geq \lambda u\left(z^{\prime}\right)+(1-\lambda) u\left(z^{\prime \prime}\right)$. We start out by showing this is true for rational $\lambda$. We construct a sequence of equally spaced points starting at $z^{\prime}$ and going up to $z^{\prime \prime}$ such that $\lambda z^{\prime}+(1-\lambda) z^{\prime \prime}$ is one of those points. We know from NII that the weighted sum of the differences prior to $\lambda z^{\prime}+(1-\lambda) z^{\prime \prime}$ is greater than the weighted sum of the differences subsequent to $\lambda z^{\prime}+(1-\lambda) z^{\prime \prime}$ and following some algebraic manipulation this gives us concavity. To deal with irrational $\lambda$ we suppose that concavity is violated for some $\hat{\lambda}$ so that $u(c)=u\left(\hat{\lambda} z^{\prime}+(1-\hat{\lambda}) z^{\prime \prime}\right)<\hat{\lambda} u\left(z^{\prime}\right)+(1-\hat{\lambda}) u\left(z^{\prime \prime}\right)$ and call $\hat{\lambda} u\left(z^{\prime}\right)+(1-\hat{\lambda}) u\left(z^{\prime \prime}\right)-u(c)$ unity. Hence, for any rational $\hat{r}$ however close to $\hat{\lambda}, u\left(\hat{r} z^{\prime}+(1-\hat{r}) z^{\prime \prime}\right)-u(c) \geq 1$ but this is impossible - because of NII, each step along the direction $(\hat{\lambda}-\hat{r}) z^{\prime}+(\hat{r}-\hat{\lambda}) z^{\prime \prime}$ will "cost" an increasing amount of value, and so we can find a point arbitrarily close to $c$ which is worse than $\mathbf{0}$ which is a contradiction to weak monotonicity. Proof. The necessary part is straightforward. Suppose $u$ is concave and consider a pair of outcomes $z^{\prime}, z^{\prime \prime} \in Z$. Then, by the definition of concavity, for $\lambda \in(0,1)$ :

$$
\begin{aligned}
u\left(z^{\prime \prime}+\lambda\left(z^{\prime}-z^{\prime \prime}\right)\right) & \geq \lambda u\left(z^{\prime}\right)+(1-\lambda) u\left(z^{\prime \prime}\right) \Rightarrow \\
u\left(z^{\prime \prime}+\lambda\left(z^{\prime}-z^{\prime \prime}\right)\right)-u\left(z^{\prime \prime}\right) & \geq \lambda\left(u\left(z^{\prime}\right)-u\left(z^{\prime \prime}\right)\right) \Rightarrow \\
u\left(z^{\prime \prime}+\lambda\left(z^{\prime}-z^{\prime \prime}\right)\right)-u\left(z^{\prime \prime}\right)-u\left(z^{\prime}\right) & \geq-\left(-\lambda u\left(z^{\prime}\right)+\lambda u\left(z^{\prime \prime}\right)+u\left(z^{\prime}\right)\right) \Rightarrow \\
u\left(z^{\prime \prime}+\lambda\left(z^{\prime}-z^{\prime \prime}\right)\right)-u\left(z^{\prime \prime}\right)-u\left(z^{\prime}\right) & \geq-\left((1-\lambda) u\left(z^{\prime}\right)+\lambda u\left(z^{\prime \prime}\right)\right) \Rightarrow \\
u\left(z^{\prime \prime}+\lambda\left(z^{\prime}-z^{\prime \prime}\right)\right)-u\left(z^{\prime \prime}\right)-u\left(z^{\prime}\right) & \geq-u\left(z^{\prime \prime}+(1-\lambda)\left(z^{\prime}-z^{\prime \prime}\right)\right) \Rightarrow \\
u\left(z^{\prime \prime}+\lambda\left(z^{\prime}-z^{\prime \prime}\right)\right)-u\left(z^{\prime \prime}\right) & \geq u\left(z^{\prime}\right)-u\left(z^{\prime \prime}+(1-\lambda)\left(z^{\prime}-z^{\prime \prime}\right)\right) .
\end{aligned}
$$

Therefore we obtain $\left(z^{\prime \prime}+\lambda\left(z^{\prime}-z^{\prime \prime}\right) \leftarrow z^{\prime \prime}\right) \succsim^{*}\left(z^{\prime} \leftarrow z^{\prime \prime}+(1-\lambda)\left(z^{\prime}-z^{\prime \prime}\right)\right)$ which represents the condition of Definition 2. The sufficiency part is a bit more complicated and we deal with it as follows. Suppose that NII holds. Consider again a pair of outcomes $z^{\prime}, z^{\prime \prime} \in Z$, and assume with no loss of generality that $z^{\prime} \succsim z^{\prime \prime}$. In the first instance we will show that concavity holds for a rational choice of $\lambda$, which we denote by $\bar{\lambda}$, i.e. $u\left(\bar{\lambda} z^{\prime}+(1-\bar{\lambda}) z^{\prime \prime}\right) \geq \bar{\lambda} u\left(z^{\prime}\right)+(1-\bar{\lambda}) u\left(z^{\prime \prime}\right)$, for $\bar{\lambda} \in(0,1)$ and $\bar{\lambda} \in \mathbb{Q}$. Since $(1-\bar{\lambda}) \in \mathbb{Q}$, there exist positive integers $\sigma$ and $\tau$, with $\sigma<\tau$, such that $(1-\bar{\lambda})=\frac{\sigma}{\tau}$. Define $\rho=\tau-\sigma$. Now consider a sequence of $\tau+1$ points $p_{k}=z^{\prime}+\frac{k}{\tau}\left(z^{\prime \prime}-z^{\prime}\right)$, $k=0, \ldots, \tau$ (so that $p_{0}=z^{\prime}, p_{\tau}=z^{\prime \prime}, p_{\sigma}=\bar{\lambda} z^{\prime}+(1-\bar{\lambda}) z^{\prime \prime}$ ). Accordingly, define the following sequence of $\tau$ terms:

$$
\begin{array}{cc}
\left(P_{1}\right) & u\left(p_{1}\right)-u\left(z^{\prime}\right) \\
\left(P_{2}\right) & u\left(p_{2}\right)-u\left(p_{1}\right) \\
\vdots & \vdots \\
\left(P_{\sigma}\right) & u\left(\bar{\lambda} z^{\prime}+(1-\bar{\lambda}) z^{\prime \prime}\right)-u\left(p_{\sigma-1}\right) \\
\left(P_{\sigma+1}\right) & u\left(p_{\sigma+1}\right)-u\left(\bar{\lambda} z^{\prime}+(1-\bar{\lambda}) z^{\prime \prime}\right) \\
\vdots & \vdots \\
\left(P_{\tau}\right) & u\left(p_{\tau}\right)-u\left(p_{\tau-1}\right) .
\end{array}
$$

By construction $p_{k}=\frac{1}{2}\left(p_{k-1}+p_{k+1}\right) \forall k=1, \ldots \tau-1$, therefore, from NII, $\left(P_{1}\right) \geq\left(P_{2}\right) \geq \ldots \geq\left(P_{\tau}\right)$, so that we can obtain the $\tau$ inequalities: $\left(P_{i}\right) \geq\left(P_{\sigma}\right), i=1, \ldots, \sigma$ and $\left(P_{i}\right) \leq\left(P_{\sigma}\right) i=\sigma+1, \ldots, \tau$. By 
summing the first $\sigma$ and the last $\rho$ inequalities separately we obtain:

$$
\begin{aligned}
& u\left(\bar{\lambda} z^{\prime}+(1-\bar{\lambda}) z^{\prime \prime}\right)-u\left(z^{\prime}\right) \geq \sigma\left(u\left(p_{\sigma}\right)-u\left(p_{\sigma-1}\right)\right) \\
& u\left(z^{\prime \prime}\right)-u\left(\bar{\lambda} z^{\prime}+(1-\bar{\lambda}) v\right) \leq \rho\left(u\left(p_{\sigma}\right)-u\left(p_{\sigma-1}\right)\right) .
\end{aligned}
$$

Dividing these by $\sigma=(1-\bar{\lambda}) \tau$ and $\rho=\bar{\lambda} \tau$ respectively gives:

$$
\begin{gathered}
\frac{1}{(1-\bar{\lambda}) \tau}\left(u\left(\bar{\lambda} z^{\prime}+(1-\bar{\lambda}) z^{\prime \prime}\right)-u\left(z^{\prime}\right)\right) \geq\left(u\left(p_{\sigma}\right)-u\left(p_{\sigma-1}\right)\right) \\
\frac{1}{\bar{\lambda} \tau}\left(u\left(z^{\prime \prime}\right)-u\left(\bar{\lambda} z^{\prime}+(1-\bar{\lambda}) v\right)\right) \leq\left(u\left(p_{\sigma}\right)-u\left(p_{\sigma-1}\right)\right),
\end{gathered}
$$

and by combining and simplifying we obtain: $u\left(\bar{\lambda} z^{\prime}+(1-\bar{\lambda}) z^{\prime \prime}\right) \geq \bar{\lambda} u\left(z^{\prime}\right)+(1-\bar{\lambda}) u\left(z^{\prime \prime}\right)$ as required. It remains to show that the Theorem also holds true for any irrational $\hat{\lambda}$, i.e. for any $z^{\prime}, z^{\prime \prime} \in Z, \hat{\lambda} \in(0,1), \hat{\lambda} \notin \mathbb{Q}$. We shall assume the contrary, i.e. that $u\left(\hat{\lambda} z^{\prime}+(1-\hat{\lambda}) z^{\prime \prime}\right)<$ $\hat{\lambda} u\left(z^{\prime}\right)+(1-\hat{\lambda}) u\left(z^{\prime \prime}\right)$ for some $z^{\prime}, z^{\prime \prime} \in Z$. We shall also assume that $z^{\prime} \succsim z^{\prime \prime}$, and that $u(\mathbf{0})=0$, with no loss of generality. Note that for any $\varepsilon>0$, we can always find a rational $\hat{r}$ such that $|\hat{r}-\hat{\lambda}|<\varepsilon$. Consider $\hat{r} \in \mathbb{Q}$, such that $\hat{r} \in(0,1)$ and $\hat{r}>\hat{\lambda}$ and define $d^{\hat{r}}=\hat{r} z^{\prime}+(1-\hat{r}) z^{\prime \prime}$, $c=\hat{\lambda} z^{\prime}+(1-\hat{\lambda}) z^{\prime \prime}$. Given that $u$ is cardinal and also because $\hat{\lambda} u\left(z^{\prime}\right)+(1-\hat{\lambda}) u\left(z^{\prime \prime}\right)-u(c)>0$ we may assume that $\hat{\lambda} u\left(z^{\prime}\right)+(1-\hat{\lambda}) u\left(z^{\prime \prime}\right)-u(c)=1$. Because $\hat{r}>\hat{\lambda}$ and $u\left(z^{\prime}\right) \geq u\left(z^{\prime \prime}\right)$, it follows that $\hat{r} u\left(z^{\prime}\right)+(1-\hat{r}) u\left(z^{\prime \prime}\right) \geq \hat{\lambda} u\left(z^{\prime}\right)+(1-\hat{\lambda}) u\left(z^{\prime \prime}\right)$, and so, because $\hat{r} \in \mathbb{Q}$, from the preceding part of the proof we obtain:

$$
\begin{aligned}
u\left(d^{\hat{r}}\right)=u\left(\hat{r} z^{\prime}+(1-\hat{r}) z^{\prime \prime}\right) & \geq \hat{r} u\left(z^{\prime}\right)+(1-\hat{r}) u\left(z^{\prime \prime}\right) \Rightarrow \\
u\left(d^{\hat{r}}\right) & \geq \hat{\lambda} u\left(z^{\prime}\right)+(1-\hat{\lambda}) u\left(z^{\prime \prime}\right) \Rightarrow \\
u\left(d^{\hat{r}}\right)-u(c) & \geq 1 .
\end{aligned}
$$

Let $\pi \in \mathbb{Z}_{+}$, consider a sequence of $\pi+1$ points and define a point $p_{k}^{\hat{r}}=d^{\hat{r}}+k\left(c-d^{\hat{r}}\right) k=0, \ldots, \pi$. Clearly it is always the case that $p_{k}^{\hat{r}} \in Z$ for any $\hat{r} \in(0,1)$ and $k=0,1$. Because $\hat{r}$ can be chosen so that it is arbitrarily close to $\hat{\lambda}$, it follows that it can be chosen such that the components of the vector $\left(c-d^{\hat{r}}\right)$ are arbitrarily close to zero. Recall that $\hat{\lambda} \in(0,1)$, i.e. $c$ is an interior point of the line segment adjoining $z^{\prime}$ and $z^{\prime \prime}$ and therefore an interior point of $Z$ since $Z$ is by assumption convex. It follows that $\hat{r}$ can be chosen such that the vector $\left(c-d^{\hat{r}}\right)$ is "small enough" so that adding $\pi$ multiples of it to $d^{\hat{r}}$ will still give a point on the line segment adjoining $z^{\prime}$ and $z^{\prime \prime}$, i.e. $\hat{r}$ can be chosen so that $p_{\pi}^{\hat{r}} \in Z$ for any given integer $\pi$. Let $\pi=\left\lceil u\left(d^{\hat{r}}\right)\right\rceil+1$. We now construct the following sequence of $\pi$ terms:

$$
\begin{array}{cc}
\left(P_{1}^{\prime}\right) & u(c)-u\left(d^{\hat{r}}\right) \\
\left(P_{2}^{\prime}\right) & u\left(p_{2}^{\hat{r}}\right)-u(c) \\
\vdots & \vdots \\
\left(P_{\pi}^{\prime}\right) & u\left(p_{\pi}^{\hat{r}}\right)-u\left(p_{\pi-1}^{\hat{r}}\right) .
\end{array}
$$

Note that, as before, $p_{k}^{\hat{r}}=\frac{1}{2}\left(p_{k-1}^{\hat{r}}+p_{k+1}^{\hat{r}}\right) \forall k=1, \ldots \pi-1$, and therefore because of NII we obtain $\left(P_{1}^{\prime}\right) \geq\left(P_{2}^{\prime}\right) \geq \ldots \geq\left(P_{\pi}^{\prime}\right)$. Recall that $u\left(d^{\hat{r}}\right)-u(c) \geq 1$, and therefore we obtain $\pi$ inequalities $\left(P_{i}^{\prime}\right) \leq-1, i=1, \ldots, \pi$, which we then sum to obtain $u\left(p_{\pi}^{\hat{r}}\right)-u\left(d^{\hat{r}}\right) \leq-\pi$ and because $\pi>u\left(d^{\hat{r}}\right)$, it follows that $u\left(p_{\pi}^{\hat{r}}\right) \leq u\left(d^{\hat{r}}\right)-\pi<0=u(\mathbf{0}) \Rightarrow \mathbf{0} \succ p_{\pi}^{\hat{r}} \in Z$, which contradicts weak-monotonicity of preferences (recall that $Z \subseteq \mathbb{R}_{+}^{p}$ ). This completes the proof. 


\section{C.2 Theorem 4}

The proof strategy for Theorem 4 is as follows. The first part of the proof considers a value assignment $v \in \mathrm{Y}_{\mathcal{C N}}$ and constructs a concave and nondecreasing value function $U_{v}(\cdot)$ that reproduces $v$, i.e. that $U_{v}\left(z_{j}\right)=v_{j} \forall j \in J$. Given a concave value function $u(\cdot)$, the second part of the proof uses a duality approach to construct a hyperplane for each $z_{k} \in R$ which passes through the point $\left(u\left(z_{k}\right), z_{k}\right)$, and above $\left(u\left(z_{j}\right), z_{j}\right)$ for all $z_{j} \in R: z_{j} \neq z_{k}$ - these correspond to the constraints of $Y_{C N}$ listed in equation (2), thus ensuring that every value assignment according to a concave value function respecting expressed preferences does indeed lie in the set in question. Proof. To begin, define for each $v \in \mathrm{Y}_{C N}$, a polyhedron $\mathbf{H}(v)$ and an associated function $U_{v}(\cdot): Z \rightarrow \mathbb{R}_{+}$as below; with these in place we split the proof in parts (i) and (ii):

$$
\begin{gathered}
\mathbf{H}(v)=\left\{(\hat{v}, \hat{z}) \in \mathbb{R}_{+}^{p+1} \mid \sum_{j} \lambda_{j} z_{j} \leq \hat{z}, \sum_{j} \lambda_{j} v_{j} \geq \hat{v}, \sum_{j} \lambda_{j}=1, \text { where } \lambda \in \mathbb{R}_{+}^{n}\right\}, \\
U_{v}(z)=\max \{\hat{v} \mid(\hat{v}, z) \in \mathbf{H}(v)\} .
\end{gathered}
$$

In part (i) we will show that the function $U_{v}(\cdot)$ is concave and nondecreasing and that $U_{v}\left(z_{j}\right)=$ $v_{j} \forall j \in J$, i.e. that $U_{v}(\cdot) \in \mathcal{U}_{\mathcal{C N}}\left(\succsim_{R}^{*}\right)$ (recall that $v$ satisfies the first set of constraints in (2) ensuring compatibility with $\left.\succsim_{R}^{*}\right)$. To show that $U_{v}(\cdot)$ is nondecreasing, consider $z^{\prime}, z^{\prime \prime} \in Z: z^{\prime \prime} \geq z^{\prime}$. From the definition of $\mathbf{H}(v)$ we obtain $\left(U_{v}\left(z^{\prime}\right), z\right)^{\prime} \in \mathbf{H}(v) \Rightarrow\left(U_{v}\left(z^{\prime}\right), z^{\prime \prime}\right) \in \mathbf{H}(v)$ and so it follows immediately from the definition of $U_{v}(\cdot)$ that $U_{v}\left(z^{\prime \prime}\right) \geq U_{v}\left(z^{\prime}\right)$. Observe that $\mathbf{H}(v)$ is by construction the hypograph of $U_{v}(\cdot)$ and since it is obviously convex, concavity of $U_{v}(\cdot)$ follows. It remains to show that $U_{v}\left(z_{j}\right)=v_{j} \forall j \in J$. Obviously, $U_{v}\left(z_{j}\right) \geq v_{j}$ (since $U_{v}(\cdot)$ is defined by a maximization operator and $\left.\left(v_{j}, z_{j}\right) \in \mathbf{H}(v)\right)$, so it is only left to show that $U_{v}\left(z_{j}\right) \leq v_{j} \forall j \in J$. Consider an arbitrary $k \in J$. Denote by $\left(U_{v}\left(z_{k}\right), \lambda^{*}\right) \in \mathbb{R}_{+}^{n+1}$ the optimal solution to the linear programming problem in (6) and let $B \subseteq J$ contain the indices of points in the reference set for which the corresponding variables $\lambda_{j}^{*}$ are in the optimal basis. By virtue of optimality the following obviously hold: (a) $U_{v}\left(z_{k}\right)=\sum_{j \in B} \lambda_{j}^{*} v_{j}$, and (b) $z_{k} \geq \sum_{j \in B} \lambda_{j}^{*} z_{j}$. By assumption $v \in \mathrm{Y}_{\mathcal{C N}}$ and therefore (from the definition of $Y_{\mathcal{C N}}$ in (2)) it holds that $v_{j}-w^{k} z_{j} \leq \beta^{k} \forall j \in B$ and also that $v_{k}-w^{k} z_{k}=\beta^{k}$. Recall that $\lambda_{j}^{*} \geq 0$ and $\sum_{j \in B} \lambda_{j}^{*}=1$, so that combining these inequalities using multipliers $\lambda_{j}^{*}$ in a weighted sum gives:

$$
\begin{aligned}
\sum_{j \in B} \lambda_{j}^{*} v_{j}-w^{k} \sum_{j \in B} \lambda_{j}^{*} z_{j} & \leq \beta^{k} \sum_{j \in B} \lambda_{j}^{*} \leq \beta^{k}=v_{k}-w^{k} z_{k} \Rightarrow \\
\sum_{j \in B} \lambda_{j}^{*} v_{j}-w^{k} \sum_{j \in B} \lambda_{j}^{*} z_{j} & \leq v_{k}-w^{k} z_{k} \Rightarrow \\
v_{k}-\sum_{j \in B} \lambda_{j}^{*} v_{j} & \geq w^{k}\left(z_{k}-\sum_{j \in B} \lambda_{j}^{*} z_{j}\right) \Rightarrow(\text { from (a) and (b)) } \\
v_{k} & \geq U_{v}\left(z_{k}\right), \text { and this completes the proof of part (i). }
\end{aligned}
$$

For part (ii) of the proof we shall consider $u(\cdot) \in \mathcal{U}_{\mathcal{C N}}(\mathrm{Y})$ and denote the value assignment according to $u(\cdot)$ as $u=\left(u\left(z_{1}\right), \ldots, u\left(z_{n}\right)\right)$. We need to show that $u \in \mathrm{Y}_{\mathcal{C N}}$. Using $u$ we can define $\mathbf{H}(u)$ and $U_{u}(\cdot)$ accordingly. We shall first show that $U_{u}\left(z_{j}\right)=u_{j} \forall j \in J$ and use this in the ensuing. As pointed out in the proof of part (i), $\left(u_{j}, z_{j}\right) \in \mathbf{H}(u) \forall j \in J$, and so it follows directly from 
the definition of $U_{u}(\cdot)$ that $U_{u}\left(z_{j}\right) \geq u_{j} \forall j \in J$. As before, we now consider an arbitrary $k \in J$, denote the corresponding optimal solution to (6) by $\left(U_{u}\left(z_{k}\right), \lambda^{*}\right) \in \mathbb{R}_{+}^{n+1}$ and also define $B \subseteq J$ in exactly the same way as in the preceding part of the proof. Therefore we obtain the following: (c) $U_{u}\left(z_{k}\right)=\sum_{j \in B} \lambda_{j}^{*} u_{j},(\mathrm{~d}) z_{k} \geq \sum_{j \in B} \lambda_{j}^{*} z_{j}$. By assumption $u(\cdot)$ is nondecreasing and so it follows from (d) that: (e) $u_{k} \geq u\left(\sum_{j \in B} \lambda_{j}^{*} z_{j}\right)$. We now combine these statements with the concavity of $u(\cdot)$ as follows:

$$
\begin{aligned}
u\left(\sum_{j \in B} \lambda_{j}^{*} z_{j}\right) & \geq \sum_{j \in B} \lambda_{j}^{*} u_{j} \Rightarrow(\text { from }(\mathrm{c})) \\
u\left(\sum_{j \in B} \lambda_{j}^{*} z_{j}\right) & \geq U_{u}\left(z_{k}\right) \Rightarrow(\text { from (e)) } \\
u_{k} & \geq U_{u}\left(z_{k}\right), \text { and this collectively gives } U_{u}\left(z_{j}\right)=u_{j} \forall j \in J .
\end{aligned}
$$

By definition, $u$ satisfies the first set of constraints in (2) ensuring compatibility with $\succsim_{R}^{*}$. Therefore we can show that $u \in \mathrm{Y}_{\mathcal{C N}}$ by demonstrating that for any $k \in J$ there will exist $w^{k}$ and $\beta^{k}$ such that the vector $\left(u, w^{k}, \beta^{k}\right)$ satisfies the corresponding conditions in (2). For any $z_{k} \in R$, consider the following linear program:

$$
\begin{array}{cc}
\max & s \\
-s+\sum_{j \in J} \lambda_{j} u_{j}=u_{k} & \left(v^{k}\right) \\
\sum_{j \in J} \lambda_{j} z_{j} \leq z_{k} & \left(w^{k}\right) \\
\sum_{j \in J} \lambda_{j}=1 & \left(\beta^{k}\right) \\
\lambda \in \mathbb{R}_{+}^{n}, s_{k} \in \mathbb{R} . &
\end{array}
$$

We associate dual variables as shown (in parentheses) so that dualizing and simplifying gives:

$$
\begin{gathered}
\min -u_{k}+w^{k} z_{k}+\beta^{k} \\
u_{j} \leq w^{k} z_{j}+\beta^{k} \forall j \in J \\
w^{k} \in \mathbb{R}_{+}^{n}, \beta^{k} \in \mathbb{R} .
\end{gathered}
$$

Let $\left(s^{*}, \lambda^{*}\right)$ denote an optimal solution to the primal and $\left(w^{k^{*}}, \beta^{k^{*}}\right)$ an optimal solution to the dual. Clearly, a solution in which $\lambda_{k}^{*}=1$ and all other variables are equal to zero is a feasible solution to the primal and therefore it must be that $s^{*} \geq 0$. However, if $s^{*}>0$ then that would imply that $\left(u_{j}+s^{*}, z_{j}\right) \in \mathbf{H}(u)$ and since we have already shown that $U_{u}\left(z_{k}\right)=u_{k}$ this gives $\left(U_{u}\left(z_{k}\right)+s^{*}, z_{j}\right) \in \mathbf{H}(u)$ which contradicts the definition of $U_{u}\left(z_{k}\right)$. Therefore $s^{*}=0$ and so duality gives $-u_{k}+w^{k^{*}} z_{k}+\beta^{k^{*}}=0$. Therefore we have demonstrated that there exist $w^{k} \in \mathbb{R}_{+}^{n}$ and $\beta^{k} \in \mathbb{R}$ such that $u_{k}=w^{k^{*}} z_{k}+\beta^{k *}$ and $u_{j} \leq w^{k} z_{j}+\beta^{k}$, and this completes part (ii) of the proof.

\section{C.3 Theorem 5}

The proof strategy for Theorem 5 is as follows. Intuitively, at each iteration more preferences are introduced and the incumbent can only "improve". Therefore $A_{t}^{*}$, the optimal value of problem 
(3), must decline and since the incumbent is always a feasible solution this value will always be nonnegative. It is then observed that $A_{t}^{*}$ is actually an upper bound on the "true" value difference between a most preferred alternative and the current incumbent. So if $A_{t}^{*}$ converges to 0 then the result is proved. If $A_{t}^{*}$ does not converge to zero, then in the case where the number of alternatives is finite, the procedure will eventually run out of alternatives which could be potentially preferred to the incumbent - so the value will effectively reach 0 at which point a most preferred alternative has been identified. In the case where the number of alternatives are infinite, if $A_{t}^{*}$ converged to a value greater than 0 (say $\Delta$ ), this would provide a bound on how far apart this infinite number of alternatives could be, but as the alternatives have to be contained in a set of finite volume, this is a contradiction and so the result is proved. Proof. Recall that $\dot{u}(\cdot): Z \rightarrow \mathbb{R}_{+}$is the "true" value function representing $\succsim^{*}$. First we establish that $\dot{u}^{\max }=\max _{z \in F(X)} \dot{u}(z)$ is well-defined. We have assumed that $Z=\mathbb{R}_{+}^{p}$ and that $\exists r>0: z \geq r \mathbf{1} \forall z \in F(X)$. Therefore $F(X)$ is contained in the interior of $Z$. The value function $\dot{u}(\cdot)$ is concave, hence continuous in the interior of its domain, so $\dot{u}(\cdot)$ is continuous over $F(X)$. We have also assumed that $X$ and hence $F(X)$ is compact. Therefore by the Weierstrass theorem the restriction of $\dot{u}(\cdot)$ to $F(X) \subset Z$ attains its maximum on $F(X)$, i.e. $u^{\max }$ exists and is finite. In the ensuing we set $r=1$, but it is easy to see that this does not restrict generality. The next part of the proof will show that as $t \rightarrow \infty, A_{t}^{*}$ converges to a nonnegative $\Delta$. For brevity, denote by Alt $\left[\succsim_{t}^{*}\right]$ the instance of problem (3) solved at iteration $t$. We first show that at every iteration $t$, problem Alt $\left[\succsim_{t}^{*}\right]$ is feasible and bounded, and that $A_{t}^{*} \geq 0$. We demonstrate feasibility by constructing a feasible solution $\dot{\gamma}=\left(\dot{v}_{0}, \dot{v}, \dot{w}^{1}, \ldots, \dot{w}^{n}, \dot{\beta}^{1}, \ldots, \dot{\beta}^{n}, \dot{x}, \dot{z}_{0}\right)$ (note that we will we use this solution again later in the proof). The preference information in $\succsim_{t}^{*}$ over $R_{t}$ is assumed to be consistent with $\succsim^{*}$ so, by Theorem 4, the value assignment of the true value function $\dot{u}(\cdot)$ which represents $\succsim^{*}$ must be recoverable through $\mathrm{Y}_{\mathcal{C N}}$, i.e. $\left(\dot{u}\left(z_{1}\right), \ldots, \dot{u}\left(z_{n}\right)\right)$ $\in \mathrm{Y}_{\mathcal{C N}}$. Set $\dot{v}_{j}=\dot{u}\left(z_{j}\right) \forall j \in J_{t}$, so that $\exists\left(\dot{v}_{j}, \dot{w}^{j}, \dot{\beta}^{j}\right) \forall j \in J_{t}$, which collectively satisfy the constraints that stipulate $v \in \mathrm{Y}_{\mathcal{C N}}$. It is easy to see that we could then pick any $\dot{x} \in X$ and use it to calculate $\dot{z}_{o}=F(\dot{x})$ and $\dot{v}_{o}=\min _{j \in I_{t}}\left\{\dot{w}^{j} \dot{z}_{o}+\dot{\beta}^{j}\right\}$ so that $\dot{\gamma}$ constitutes a feasible solution to $\mathbf{A l t}\left[\succsim_{t}^{*}\right]$. To show that Alt $\left[\succsim_{t}^{*}\right]$ is bounded, observe first that for $z_{1}=\mathbf{0}$ it holds that $v_{1}=0$ and so from the constraints defining $\mathrm{Y}_{\mathcal{C N}}$ we obtain $0=v_{1} \leq w^{j} \mathbf{0}+\beta^{j} \forall j \in J \Rightarrow \beta^{j} \geq 0 \forall j \in J$. Similarly, for $z_{2}=\mathbf{1}$ it holds that $1=v_{2}=w^{2} \mathbf{1}+\beta^{2}$ and since $w^{2}, \beta^{2} \geq 0$, it follows that the values of $w^{2}$ and $\beta^{2}$ are bounded. Since $v_{0} \leq w^{2} z_{j}+\beta^{2}$ it follows that the optimal objective function value is bounded. To show that $A_{t}^{*} \geq 0$ we consider separately the first iteration from all subsequent iterations. At iteration $t=1$, no preference information is known, i.e. $\succsim_{1}^{*}=\varnothing$, and $R_{1}=\{\mathbf{0}, \mathbf{1}\}$. So at this stage $\mathcal{U}_{\mathcal{C N}}(\mathrm{Y})$ contains any concave nondecreasing value function $u(\cdot)$ such that $u(\mathbf{0})=0$ and $u(\mathbf{1})=1$. We have assumed that $\succsim$ is weakly monotonic and also that $z \geq \mathbf{1}, \forall z \in F(X)$. Therefore $z \succsim \mathbf{1}, \forall z \in F(X)$ which implies $u(z) \geq 1 \forall z \in F(X)$. Therefore, $\forall z \in F(X)$ and $u(\cdot) \in \mathcal{U}_{\mathcal{C N}}(\mathrm{Y}): u(z)-u(\mathbf{1})=u(z)-1 \geq 0$, and so $A_{1}^{*}=v_{0}^{*}-1 \geq 0$. In subsequent iterations, we know of an incumbent solution $x_{t}^{i n c}$ prior to the solution of Alt $\left[\succsim_{t}^{*}\right]$. We can show that $A_{t}^{*} \geq 0$ by demonstrating the existence of a feasible solution with an associated objective function value equal to zero. Earlier we constructed a feasible solution $\dot{\gamma}=\left(\dot{v}_{0}, \dot{v}, \dot{w}^{1}, \ldots, \dot{w}^{n}, \dot{\beta}^{1}, \ldots, \dot{\beta}^{n}, \dot{x}, \dot{z}_{0}\right)$ for an arbitrary choice of $\dot{x} \in X$. So now we can set $\dot{x}=x_{t}^{i n c}$ so that $\dot{z}_{o}=z_{t}^{i n c}$ and therefore $\dot{v}_{o}=\dot{v}_{j^{\prime}}$, so the objective function value for $\dot{\gamma}$ is zero. Next we show that $A_{t+1}^{*} \leq A_{t}^{*}$ for any two consecutive iterations $t$ and $t+1$. To distinguish the optimal values of different variables between iterations $t$ and $t+1$ we use $v_{t}^{i n c}, v_{t+1}^{i n c}$ for the optimal value assignment to the incumbent alternative (i.e. the optimal value $v_{j^{\prime}}^{*}$ at each iteration) and add the index $t$ to all other variables. At iteration $t$ there are two distinct possibilities for updating the expressed preference ordering: a) $z_{t}^{i n c} \succ z_{o t}^{*}$;b) $z_{o t}^{*} \succsim$ 
$z_{t}^{i n c}$. In the first case we construct $\mathbf{A l t}\left[\succsim_{t+1}^{*}\right]$ by adding an additional constraint to $\mathbf{A l t}\left[\succsim_{t}^{*}\right]$ and so $A_{t+1}^{*} \leq A_{t}^{*}$ follows immediately. In the second case, in addition to adding an additional constraint, the objective function also changes because the incumbent solution is updated to $z_{t+1}^{i n c}=z_{o t}^{*}$. The new constraint is introduced to guarantee that $u\left(z_{o t}^{*}\right)=u\left(z_{t+1}^{i n c}\right) \geq u\left(z_{t}^{i n c}\right)$ for any compatible value function $u(\cdot) \in \mathcal{U}_{\mathcal{C N}}(\mathrm{Y})$. Suppose that $A_{t+1}^{*}>A_{t}^{*}=v_{o t}^{*}-v_{t}^{i n c}$. Then there exists a value function $u(\cdot)$ compatible with $\left(\succsim_{t+1}^{*}, R_{t+1}\right)$ (and therefore also compatible with $\left.\left(\succsim_{t}^{*}, R_{t}\right)\right)$, such that:

$$
\begin{aligned}
u\left(z_{o t+1}^{*}\right)-u\left(z_{t+1}^{i n c}\right) & >v_{o t}^{*}-v_{t}^{i n c} \Rightarrow\left(\text { because } z_{t+1}^{i n c}=z_{o t}^{*}\right) \\
u\left(z_{o t+1}^{*}\right)-u\left(z_{o t}^{*}\right) & \left.>v_{o t}^{*}-v_{t}^{i n c} \Rightarrow \text { (because } z_{o t}^{*} \succsim z_{t}^{i n c}\right) \\
u\left(z_{o t+1}^{*}\right)-u\left(z_{t}^{i n c}\right) & >v_{o t}^{*}-v_{t}^{i n c},
\end{aligned}
$$

however this would be a contradiction because $A_{t}^{*}$ is the optimal value of $\mathbf{A l t}\left[\succsim_{t}^{*}\right]$. Thus we have shown that for all iterations $t, 0 \leq A_{t+1}^{*} \leq A_{t}^{*} \leq A_{1}^{*}$, i.e. the sequence of real numbers $\left\{A_{t}^{*}\right\}$ is nonincreasing and bounded from below. Therefore, by the monotone convergence theorem, it follows that as $t \rightarrow \infty, A_{t}^{*}$ converges to a value $\Delta: 0 \leq \Delta \leq A_{1}^{*}$. In fact, if $\Delta=0$ this would imply that the true value of the incumbent alternative, $\dot{u}\left(z_{t}^{i n c}\right)$, converges to the true maximal value $\dot{u}^{\max }=\max _{z \in F(X)} \dot{u}(z)$. This follows directly from the fact that the values $A_{t}^{*}$ are upper bounds for the true value difference $\dot{u}^{\max }-\dot{u}\left(z_{t}^{i n c}\right)$. To show the latter, assume that for some iteration $t$ we have $\dot{u}^{\max }-\dot{u}\left(z_{t}^{i n c}\right)>A_{t}^{*}$. Define $x^{\max } \in X, z^{\max }=F\left(x^{\max }\right)$ such that $\dot{u}\left(z^{\max }\right)=\dot{u}^{\max }$. Now we use the solution $\dot{\gamma}$ constructed as was done earlier in the proof, by setting $\dot{v}_{o}=\dot{u}\left(z^{\max }\right), \dot{x}_{o}=x^{\max }$ and $z_{0}=z^{\text {max }}$. Then $\dot{\gamma}$ would be a feasible solution to Alt $\left[\succsim_{t}^{*}\right]$ with an objective value strictly greater than $A_{t}^{*}$, which contradicts optimality. From this we obtain that $\dot{u}\left(z^{\max }\right)-\dot{u}\left(z_{t}^{i n c}\right) \leq A_{t}^{*} \forall t$. So if $A_{t}^{*}$ converges to 0 then $\dot{u}\left(z_{t}^{i n c}\right)$ converges to the true maximal value $\dot{u}^{\text {max }}$. It remains to show that as $t \rightarrow \infty, A_{t}^{*}$ converges to $\Delta=0$, which we do in the remainder of the proof. We suppose the contrary, i.e. $\Delta>0$. Recall that $z_{o t}^{*}$ denotes the challenger alternative identified at iteration $t$. Note that, because $A_{t}^{*}>0$, it follows that the challenger alternative $z_{o t}^{*}$ cannot be identical to any of the identified alternatives in the reference set $R_{t}$, as all of these are (weakly) less preferred to the incumbent alternative, i.e. $z_{o t}^{*} \neq z_{j} \forall z_{j} \in R_{t}$. Observe that if $X$ is a finite set, it is not possible to generate a new challenger at each iteration for an arbitrarily large number of iterations and we have a contradiction and are already done (in other words it is possible to explicitly consider all distinct points in finite time); otherwise, read on. At this point the reader may benefit from a structure of the remainder of the proof, which deals with the case where $X$ is an infinite set. We are aiming to falsify the supposition that $\Delta>0$ and hence there is infinite series of new challengers. To do this we construct a neighborhood around each point in the reference set such that each new challenger is outside the neighborhood of all alternatives in the reference set (supposing that the challenger is within this neighborhood leads to an arithmetic impossibility). At some point, as one proceeds down the infinite list of challengers, there will be no more room for a new challenger because the solution space is compact. We begin the construction of the neighborhood by finding a positive real $\Gamma$ such that $u(z) \leq \Gamma$, for any $z \in F(X)$ and $u(\cdot) \in \mathcal{U}_{\mathcal{C N}}$ with $u(\mathbf{0})=0$ and $u(\mathbf{1})=1$. It is easy to see that we may set $\Gamma=v_{o}^{*}$, where $v_{o}^{*}$ comes from the optimal solution to $\mathbf{A l t}\left[\succsim_{1}^{*}\right]$ at the first iteration and is the optimal value assignment to the challenger alternative. If it was the case that a different $\hat{u}(\cdot)$ existed which satisfied $\hat{u}(\mathbf{0})=0$ and $\hat{u}(\mathbf{1})=1$ so that $\hat{u}\left(z_{0}^{*}\right)>v_{0}^{*}$, then it would hold that $\hat{u}\left(z_{o}^{*}\right)-1>v_{o}^{*}-1=A_{t}^{*}$, and since $\hat{u}(\cdot)$ is be compatible with $\succsim_{1}^{*}$ this would contradict optimality of $A_{t}^{*}$. Using $\Gamma=v_{o}^{*}$ and $\Delta>0$ (as per our previous assumption) we now define a positive integer $\tau=\left\lceil\frac{\Gamma}{\Delta}\right\rceil+1$. Consider the challenger alternative $z_{o t}^{*}$, identified at 
iteration $t$ by solving $\mathbf{A l t}\left[\succsim_{t}^{*}\right]$ and let $z_{r} \in R_{t}$ be an arbitrary reference alternative. We shall now show that it cannot be the case that $z_{o t}^{*} \leq z_{r}+\mathbf{1}\left(\frac{1}{\tau-1}\right)$. Let us assume, on the contrary, that this is true and let $\bar{u}(\cdot) \in \mathcal{U}_{\mathcal{C N}}$ be a value function which reproduces the value assignment $\left(v_{0}^{*}, v_{1}^{*}, \ldots, v_{n}^{*}\right)$. Then it holds that $A_{t}^{*}=\bar{u}\left(z_{o t}^{*}\right)-\bar{u}\left(z_{t}^{i n c}\right)>0$, and since $z_{t}^{i n c} \succsim z_{j}$ for any $z_{j} \in R_{t}$, it follows that $\bar{u}\left(z_{o t}^{*}\right)>\bar{u}\left(z_{r}\right)$. As in the proof of Proposition 3, we construct a sequence of $\tau+1$ points: $q_{k}=z_{o t}^{*}-k\left(z_{o t}^{*}-z_{r}\right), k=0,1, \ldots, \tau$ so that $q_{0}=z_{o t}^{*}, q_{1}=z_{r}$ and each intermediate point in the sequence is the midpoint of its predecessor and its successor. First we show that $q_{\tau} \in \mathbb{R}_{+}^{p}$. Let $z_{o t i}^{*}$ denote the $i$-th coordinate of $z_{o t}^{*}$ and recall that we have assumed that $z_{o t}^{*} \leq z_{r}+\mathbf{1}\left(\frac{1}{\tau-1}\right)$ and also $z_{r} \geq 1$. It follows that:

$$
\begin{aligned}
q_{\tau i} & =z_{o t i}^{*}-\tau\left(z_{o t i}^{*}-z_{r i}\right) \Rightarrow \\
q_{\tau i} & =(1-\tau) z_{o t i}^{*}+\tau z_{r i} \Rightarrow \\
q_{\tau i} & \geq(1-\tau) z_{r i}+\frac{1-\tau}{\tau-1}+\tau z_{r i} \Rightarrow \\
q_{\tau i} & \geq z_{r i}-1 \geq 0 \Rightarrow \\
q_{\tau} & \in \mathbb{R}_{+}^{p} .
\end{aligned}
$$

Since, $\bar{u}(z) \geq 0 \forall z \in \mathbb{R}_{+}^{p}$ from weak monotonicity, $\bar{u}\left(q_{\tau}\right) \geq 0$. We now construct the following sequence of $\tau$ terms:

$$
\begin{array}{cc}
\left(Q_{1}\right) & \bar{u}\left(z_{r}\right)-\bar{u}\left(z_{o t}^{*}\right) \\
\left(Q_{2}\right) & \bar{u}\left(q_{2}\right)-\bar{u}\left(q_{1}\right) \\
\vdots & \vdots \\
\left(Q_{\tau}\right) & \bar{u}\left(q_{\tau}\right)-\bar{u}\left(q_{\tau-1}\right) .
\end{array}
$$

By NII it must be that $\left(Q_{i}\right) \geq\left(Q_{1}\right) \forall i=1, \ldots, \tau$, so we can add these inequalities and obtain:

$$
\begin{aligned}
\bar{u}\left(q_{\tau}\right)-\bar{u}\left(q_{0}\right) & \leq \tau\left(\bar{u}\left(q_{1}\right)-\bar{u}\left(q_{0}\right)\right) \Rightarrow \\
\bar{u}\left(q_{\tau}\right) & \leq \bar{u}\left(q_{0}\right)-\tau\left(\bar{u}\left(q_{0}\right)-\bar{u}\left(q_{1}\right)\right) \Rightarrow \\
\bar{u}\left(q_{\tau}\right) & \left.\leq \bar{u}\left(z_{o t}^{*}\right)-\tau\left(\bar{u}\left(z_{o t}^{*}\right)-\bar{u}\left(z_{r}\right)\right) \Rightarrow \text { (because } \bar{u}\left(z_{t}^{i n c}\right) \geq \bar{u}\left(z_{r}\right)\right) \\
\bar{u}\left(q_{\tau}\right) & \leq \bar{u}\left(z_{o t}^{*}\right)-\tau\left(\bar{u}\left(z_{o t}^{*}\right)-\bar{u}\left(z_{t}^{i n c}\right)\right) \Rightarrow \\
\bar{u}\left(q_{\tau}\right) & \leq \bar{u}\left(z_{o t}^{*}\right)-\tau A_{t}^{*} .
\end{aligned}
$$

But in this case observe that:

$$
\begin{aligned}
\bar{u}\left(q_{\tau}\right) & \geq 0 \Rightarrow \\
\bar{u}\left(z_{o t}^{*}\right)-\tau A_{t}^{*} & \geq 0 \Rightarrow \\
\frac{\bar{u}\left(z_{o t}^{*}\right)}{A_{t}^{*}} & \geq \tau \Rightarrow\left(\text { because } \tau=\left\lceil\frac{\Gamma}{\Delta}\right\rceil+1\right) \\
\frac{\bar{u}\left(z_{o t}^{*}\right)}{A_{t}^{*}} & \geq\left\lceil\frac{\Gamma}{\Delta}\right\rceil+1 \Rightarrow\left(\text { because } \bar{u}\left(z_{o t}^{*}\right) \leq \Gamma \text { and } A_{t}^{*} \geq \Delta\right) \\
\frac{\Gamma}{\Delta} & \geq\left\lceil\frac{\Gamma}{\Delta}\right\rceil+1,
\end{aligned}
$$


so we have our contradiction to the supposition that $z_{o t}^{*} \leq z_{r}+\mathbf{1}\left(\frac{1}{\tau-1}\right)$. Therefore if $\Delta>0$, then at every iteration $t$ we can solve $\mathbf{A l t}\left[\succsim_{t}^{*}\right]$ and identify a challenger alternative $z_{o t}^{*}$ which for each $z_{j} \in R_{t}$ satisfies $z_{o t i}^{*}>z_{j i}+\frac{1}{\tau-1}$ for at least one coordinate $i$. Let $B(z, r)$ denote the closed ball of radius $r$ centered at a point $z \in \mathbb{R}^{p}$ and consider the set of all points which can be contained by balls of radius $\frac{1}{3(\tau-1)}$ centered at points $z \in F(X)$, i.e.:

$$
\mathcal{Z}=\left\{\zeta \in \mathbb{R}^{p} \mid \zeta \in B\left(z, \frac{1}{3(\tau-1)}\right), z \in F(X)\right\}
$$

Because $F(X)$ is compact, $\mathcal{Z}$ is also compact and it has finite volume $\operatorname{vol}(\mathcal{Z})=V$. For every iteration $t$, define a closed ball $B_{t}=B\left(z_{o t}^{*}, \frac{1}{3(\tau-1)}\right)$ with volume $\delta=\operatorname{volume}\left(B_{t}\right) \forall t$. Since $z_{o t i}^{*} \not \leq z_{j i}+\frac{1}{\tau-1} \forall z_{j} \in R_{t}$, it follows that $d\left(z_{o t}^{*}, z_{j}\right) \geq \frac{1}{\tau-1} \forall z_{j} \in R_{t}$ (where $d(\cdot, \cdot)$ denotes the usual Euclidean distance metric). So any closed ball around $z_{o t}^{*}$ with radius $r$ strictly smaller than $\frac{1}{2(\tau-1)}$ (greater than half of any distance $\left.d\left(z_{o t}^{*}, z_{j}\right)\right)$ cannot contain any reference alternative $z_{j} \in R_{t}$. Since $z_{o t}^{*}$ will be added to the set of reference alternatives in iteration $t+1$, it follows that successive balls $B_{t}$ must be disjoint. Therefore, for any iteration $T$ we have: volume $\left(\bigcup_{t=1 \ldots T} B_{t}\right)$ $=\sum_{t=1 \ldots T} \operatorname{volume}\left(B_{t}\right)=\delta T$. By construction, however, $B_{t} \subseteq \mathcal{Z} \forall t \Rightarrow \bigcup_{t=1 \ldots T} B_{t} \subseteq \mathcal{Z}$ and so volume $\left(\bigcup_{t=1 \text { to } T} B_{t}\right) \leq \operatorname{volume}(\mathcal{Z}) \Leftrightarrow \delta T \leq V$. However, we can choose $T$ as high as we like and thus secure a contradiction. Therefore it must be true that $\Delta=0$ and this completes the proof.

\section{References}

Glover, F. 1975. Improved linear integer programming formulations of nonlinear integer programs. Management Science 22(4) 455-460. 